\title{
Dynamical Analysis of the Lorenz-84 Atmospheric Circulation Model
}

\author{
Hu Wang, Yongguang Yu, and Guoguang Wen \\ Department of Mathematics, Beijing Jiaotong University, Beijing 100044, China \\ Correspondence should be addressed to Yongguang Yu; ygyu@bjtu.edu.cn
}

Received 3 July 2014; Revised 15 September 2014; Accepted 16 September 2014; Published 23 November 2014

Academic Editor: Qingdu Li

Copyright (C) $2014 \mathrm{Hu}$ Wang et al. This is an open access article distributed under the Creative Commons Attribution License, which permits unrestricted use, distribution, and reproduction in any medium, provided the original work is properly cited.

The dynamical behaviors of the Lorenz-84 atmospheric circulation model are investigated based on qualitative theory and numerical simulations. The stability and local bifurcation conditions of the Lorenz-84 atmospheric circulation model are obtained. It is also shown that when the bifurcation parameter exceeds a critical value, the Hopf bifurcation occurs in this model. Then, the conditions of the supercritical and subcritical bifurcation are derived through the normal form theory. Finally, the chaotic behavior of the model is also discussed, the bifurcation diagrams and Lyapunov exponents spectrum for the corresponding parameter are obtained, and the parameter interval ranges of limit cycle and chaotic attractor are calculated in further. Especially, a computerassisted proof of the chaoticity of the model is presented by a topological horseshoe theory.

\section{Introduction}

Atmospheric models provided an excellent instrument for complex dynamical behaviors which can be observed in natural science. They involve processes occurring over a wide spectrum of space scales and time scales, from the chemistry of minor constituents in the stratosphere to hurricanes, droughts, or the Quaternary glaciations, and give rise to a variety of intricate behaviors in the form of abrupt transitions, wave propagation, weak chaos, or fully developed turbulence [1-7]. The generally accepted approaches to study the atmospheric and climate dynamics are based on numerical forecasting models, in which all processes deemed to be relevant are included. As for the low-order atmospheric models, they involve a large number equations. Although it is unreasonable to expect solutions to low-dimensional problems to generalize to a million-dimensional spaces, it is unlikely too that problems identified in the simplified models will vanish in operational models [8]. It is equally important to note recent results which indicate the possibility that high-dimensional models may behave in a smooth way with respect to changes in parameter values [9-11]. Thus, low-order models may well have little to do with higherdimensional operational models.
On the other side, the earth's atmosphere is in constant circulation due to the earth's atmosphere being heated by the sun and the earth's rotation. A succession of the heating of the air near the earth's surface, rising, and cool air coming down sets up a general circulation pattern: air rises near the equator, moves north and south away from the equator at higher altitudes, sinks down near the poles, and flows back along the surface from both poles to the equator [12]. This important type of flow is called Hadley circulation that was first named after Hadley [13]. There is some evidence that the expansion of the Hadley circulation is related to climate change [14]. The majority of earth's driest and arid regions are located in the areas underneath the descending branches of the Hadley circulation around 30 degrees latitude. Both idealised and more realistic climate model experiments show that the Hadley circulation expands with increased global mean temperature; this can lead to large changes in precipitation in the latitudes at the edge of the cells [15]. Scientists fear that the ongoing presence of global warming might bring changes to the ecosystems in the deep tropics and that the deserts will become drier and expand [16]. Based on the above discussion, the Hadley circulation is very important to the atmospheric science. Furthermore, the stable and unstable atmospheric circulations are closely linked with the dramatic changes and 
persistent abnormalities of the weather. Therefore, it is very important to research the stable and unstable atmospheric circulation for meteorological phenomena.

A very appealing low-order model of atmospheric circulation is introduced by Lorenz in 1984 [17], which is called Lorenz-84 atmospheric circulation model (Lorenz-84 model). The Lorenz-84 model involves just three ordinary differential equations, and it includes some important features of Hadley circulation. So far, this model was known to have a pair of coexisting climates described originally by Lorenz [18]. Due to the importance of Lorenz-84 model, it has received great attention from researchers, and many important results on Lorenz-84 model have also been obtained [1923]. In 1995, Shil'nikov et al. discussed the bifurcation and predictability of the Lorenz-84 model [19]. Soon afterward, Broer et al. studied the bifurcations and strange attractors in the Lorenz-84 model with seasonal forcing [20]. Van et al. [21] and Roebber [22] investigated the dynamical behaviors of a low-order coupled ocean-atmospheric model. It is well known that the synoptic atmospheric dynamics over the North Atlantic ocean can be dominated by the jet stream, a westerly circulation, and baroclinic waves, which transport heat and momentum northward. Based on this fact, Kuznetsov et al. considered the intensity of the jet stream and discussed the fold-flip bifurcation in the Lorenz-84 model [23]. For some more detailed investigations for the Lorenz-84 model, the interested reader could also see [24-29]. However, the most important results are mainly based on numerical simulations in $[19,20,22,28]$.

This paper aims to further investigate the dynamical behaviors of the Lorenz- 84 model by theoretical analysis. Some stability conditions, supercritical, and subcritical Hopf bifurcations are obtained by using the qualitative analysis method. Moreover, bifurcation analysis of a nonlinear dynamical system throws useful light on the behavior of the system in different parameter ranges. Generally, equilibrium points play an important role in governing the overall system behavior. It is therefore useful to consider the mathematical expressions of equilibrium points as a function of system parameters. However, it is so difficult to obtain the equilibrium points regarded as the explicit mathematical expressions about the parameters for the Lorenz-84 model. In this paper, one component of equilibrium point is regarded as a parameter, and others are considered as its functions. In this way, it is not necessary to know what kind of the equilibrium point it is, and the stability conditions and the direction of the Hopf bifurcation are still obtained. In addition, there are many important results about the chaotic behaviors of the Lorenz-84 model [17-20, 22, 28], in which the chaotic behaviors of the Lorenz- 84 model were studied by considering only one parameter. In this paper, the chaotic behaviors of the Lorenz-84 model are studied by considering every parameter in the model. Furthermore, the topological horseshoe is given in the Lorenz-84 model, which provides a powerful tool in the rigorous study of chaos. Finally, some similar dynamical behaviors of the Lorenz-84 model under different parameters are founded, which will be very useful for discussing the codimension- $n(n \geq 2)$ bifurcation or other nonlinear phenomena.
This paper is arranged as follows. In Section 2, the stability and bifurcation of the model are discussed, and the conditions of stability and bifurcation are also given. Especially, the Hopf bifurcation is discussed, and the conditions of the supercritical and subcritical bifurcation are derived. In addition, some numerical simulations are shown to verify our theoretical results and conditions. The chaotic behavior of Lorenz-84 model is researched in Section 3. The bifurcation diagrams and Lyapunov exponent spectrum for every parameter are discussed, and the parameter interval ranges of limit cycle and chaotic attractor of every parameter are calculated. Conclusions in Section 4 close the paper.

\section{The Stability and Bifurcation Analysis of the Lorenz-84 Model}

In this section, we mainly discuss the stability and local bifurcation of the Lorenz-84 model and obtain the stability conditions and direction of the Hopf bifurcation.

2.1. The Lorenz-84 Model. The Lorenz-84 model is a threedimensional system [17] and is given by

$$
\begin{aligned}
& \frac{d x}{d t}=-y^{2}-z^{2}-a x+a F, \\
& \frac{d y}{d t}=x y-b x z-y+G, \\
& \frac{d z}{d t}=b x y+x z-z,
\end{aligned}
$$

where $x$ represents the strength of the globally averaged westerly current and $y$ and $z$ are the strength of the cosine and sine phases of a chain of superposed waves. The unit of the variable $t$ is equal to the damping time of the waves that is estimated to be five days. $F$ and $G$ represent the thermal forcing terms, and the parameter $b$ represents the advection strength of the waves by the westerly current. Hence, the equilibrium point of model (1) satisfies the following equation:

$$
\begin{gathered}
-y^{2}-z^{2}-a x+a F=0, \\
x y-b x z-y+G=0, \\
b x y+x z-z=0 .
\end{gathered}
$$

That is,

$$
\begin{gathered}
y=\frac{(1-x) G}{1-2 x+\left(1+b^{2}\right) x^{2}}, \\
z=\frac{b x G}{1-2 x+\left(1+b^{2}\right) x^{2}}, \\
a(F-x)\left(1-2 x+\left(1+b^{2}\right) x^{2}\right)=G^{2} .
\end{gathered}
$$

It is well known that when the parameter $G=0$, the dynamical behaviors of model (1) are simple which have been discussed in [24], while the parameter $G \neq 0$, the dynamical behaviors become complicated and display chaotic 
attractors in model (1). The objective of this paper is to discuss the stability and local bifurcation and to obtain the corresponding stability and bifurcation conditions about model (1) with $G \neq 0$. Note from (2) that it is difficult to obtain the explicit mathematical expression of the equilibrium points on the parameters $a, b, F$, and $G$. However, we can also view the variable $x$ as a parameter. Then, we can get $y=$ $y(x, a, b, F, G), z=z(x, a, b, F, G)$ and $x$ satisfies the equation $a(F-x)\left(1-2 x+\left(1+b^{2}\right) x^{2}\right)=G^{2}$, that are shown in (3).

2.2. The Stability and Bifurcation Analysis with Parameters $G \neq$ 0 and $b=0$. When $G \neq 0$ and $b=0$, model (1) is given as

$$
\begin{aligned}
& \frac{d x}{d t}=-y^{2}-z^{2}-a x+a F, \\
& \frac{d y}{d t}=x y-y+G, \\
& \frac{d z}{d t}=x z-z .
\end{aligned}
$$

Then, the equilibrium point of model (4) satisfies the following equation:

$$
\begin{gathered}
-y^{2}-z^{2}-a x+a F=0, \\
x y-y+G=0, \\
x z-z=0 .
\end{gathered}
$$

From the third equation, in (5), we have $(x-1) z=0$. If $x=1$, then, from the second equation in (5), $G=0$, which is contradictory to the hypotheses $G \neq 0$ in this section. Hence, it implies $z=0$ and $x \neq 1$. Then, we can obtain an equilibrium point $K\left(x_{0}, G /\left(1-x_{0}\right), 0\right)$ of model (1), where $x_{0}$ satisfies the equation $a(F-x)(1-x)^{2}=G^{2}$ and $x_{0} \neq 1$.

Theorem 1. When $G \neq 0$ and $b=0$, there exists at least one equilibrium point $K\left(x_{0}, G /\left(1-x_{0}\right), 0\right)$ in model (1)

(1) if $a>0$ and the equation $a(F-x)(1-x)^{2}=G^{2}$ has solution $x_{0}<1$, the equilibrium point $K$ is stable,

(2) if $a>0$ and the equation $a(F-x)(1-x)^{2}=G^{2}$ has solution $x_{0}>1$, the equilibrium point $K$ is unstable,

(3) if $a<0$ and the equation $a(F-x)(1-x)^{2}=G^{2}$ has solution $1-\sqrt[3]{-2 G^{2} / a}<x_{0}<1+a$, the equilibrium point $K$ is stable.

Proof. The Jacobian matrix of model (1) at the point $K\left(x_{0}, G /\left(1-x_{0}\right), 0\right)$ is given as

$$
J=\left(\begin{array}{ccc}
-a & -2 y_{0} & 0 \\
y_{0} & x_{0}-1 & 0 \\
0 & 0 & x_{0}-1
\end{array}\right),
$$

and its characteristic equation is given as

$$
\begin{array}{r}
f(\lambda)=\left(\lambda+1-x_{0}\right)\left(\lambda^{2}+\left(a+1-x_{0}\right) \lambda\right. \\
\left.+(a+1) x_{0}+2 y_{0}^{2}\right) .
\end{array}
$$

Then, eigenvalues $\lambda_{1}, \lambda_{2}$, and $\lambda_{3}$ are obtained as follows:

$$
\begin{aligned}
& \lambda_{1}=x_{0}-1, \\
& \lambda_{2}=\frac{-\left(a+1-x_{0}\right)+\sqrt{\left(a-1+x_{0}\right)^{2}-\left(8 G^{2} /\left(1-x_{0}\right)^{2}\right)}}{2}, \\
& \lambda_{3}=\frac{-\left(a+1-x_{0}\right)-\sqrt{\left(a-1+x_{0}\right)^{2}-\left(8 G^{2} /\left(1-x_{0}\right)^{2}\right)}}{2} .
\end{aligned}
$$

If $a>0$ and the equation $a(F-x)(1-x)^{2}=G^{2}$ has solution $x_{0}<1$, then there is $\lambda_{1}<0$. Since $8 G^{2} /\left(1-x_{0}\right)^{2}>0$ and $\left(a+1-x_{0}\right)^{2}-\left(a-1+x_{0}\right)^{2}=4\left(1-x_{0}\right) a>0$, it follows that $\operatorname{Re}\left(\lambda_{2}\right)<0$ and $\operatorname{Re}\left(\lambda_{3}\right)<0$. Therefore, the equilibrium point $K$ is stable. If the solution $x_{0}>1$, it is easy to verify that the equilibrium point $K$ is unstable. If $a<0$ and the equation $a(F-x)(1-x)^{2}=G^{2}$ has solution $1-\sqrt[3]{-2 G^{2} / a}<x_{0}<$ $1+a$, it follows that $\operatorname{Re}\left(\lambda_{1}\right)<0, \operatorname{Re}\left(\lambda_{2}\right)<0$, and $\operatorname{Re}\left(\lambda_{3}\right)<$ 0 . Therefore, the equilibrium point $K$ is stable. The proof is completed.

Theorem 2. If $G \neq 0$ and $b=0$, then there exists at least one equilibrium point $K\left(x_{0}, G /\left(1-x_{0}\right), 0\right)$ in model (1)

(1) when $a>0$ and $2 G^{2}=a^{4}$, if $x_{0}=a+1$ is a solution of the equation $a(F-x)(1-x)^{2}=G^{2}$, the equilibrium point $K\left(x_{0}, G /\left(1-x_{0}\right), 0\right)$ is unstable and model (1) has a fold bifurcation,

(2) when $a<0$ and $2 G^{2}>a^{4}$, if $x_{0}=a+1$ is a solution of the equation $a(F-x)(1-x)^{2}=G^{2}$, the equilibrium point $K\left(x_{0}, G /\left(1-x_{0}\right), 0\right)$ is unstable, and model (1) has a Hopf bifurcation.

Proof. It is easy to obtain the result from (8) and is therefore omitted here.

2.3. Direction and Stability of the Hopf Bifurcation. In this section, the supercritical and subcritical bifurcations of model (1) are considered. According to Theorem 2, model (1) has a Hopf bifurcation when $x_{0}=a+1,2 G^{2}>a^{4}$, and $a<0$. Let the eigenvectors corresponding to the eigenvalues $\lambda_{1}$ and $\lambda_{3}$ be $\alpha_{1}+i \alpha_{2}$ and $\alpha_{3}$, respectively, where $\alpha_{1}, \alpha_{2}$, and $\alpha_{3}$ are all real vectors. Under the following linear transformation:

$$
\begin{aligned}
& x_{1}=x-x_{0}, \\
& y_{1}=y-y_{0}, \\
& z_{1}=z-z_{0},
\end{aligned}
$$


model (1) can be changed into

$$
\begin{aligned}
& \dot{x}_{1}=-y_{1}^{2}-2 y_{0} y_{1}-y_{0}^{2}-z_{1}^{2}+a x_{0}-a x_{1}+a F, \\
& \dot{y}_{1}=x_{1} y_{1}+x_{1} y_{0}+x_{0} y_{1}+x_{0} y_{0}-y_{1}-y_{0}+G, \\
& \dot{z}_{1}=x_{1} z_{1}+x_{0} z_{1}-z_{1} .
\end{aligned}
$$

For (6), (7), and (8), if $x_{0}=a+1$ is a solution of the equation $a(F-x)(1-x)^{2}=G^{2}, 2 G^{2}>a^{4}$ and $a<0$, we have

$$
\begin{gathered}
\alpha^{\prime}(0)=\operatorname{Re}\left(\lambda^{\prime}(a)\right)=\frac{1}{2}>0, \\
\omega^{\prime}(0)=\operatorname{Im}\left(\lambda^{\prime}(a)\right)=\frac{\sqrt{4 G^{2}-a^{4}}}{4\left(2 G^{2}-a^{4}\right)} \neq 0, \\
\omega(0)=\operatorname{Im}(\lambda(a))=\frac{\sqrt{2 G^{2}-a^{4}}}{|a|}>0, \\
\lambda_{3}<0 .
\end{gathered}
$$$$
A=\left(\begin{array}{ccc}
0 & -k & 0 \\
k & 0 & 0 \\
0 & 0 & a
\end{array}\right), \quad \widetilde{x_{2}}=\left(\begin{array}{l}
x_{2} \\
y_{2} \\
z_{2}
\end{array}\right),
$$$$
B=\left(\begin{array}{c}
\frac{a}{k} x_{2}^{2}+x_{2} y_{2}+\frac{a k}{G} x_{0} y_{0}-y_{0}+G \\
-\frac{G^{2}+a^{4}}{a^{2} k^{2}} x_{2}^{2}-\frac{a}{k} x_{2} y_{2}-z_{2}^{2}-\left(\frac{a^{2}}{G}+\frac{2 G}{a k}\right) x_{0} y_{0}-a x_{0}+\frac{a^{2}}{G} x_{0}+a F-a^{2} \\
\frac{a}{k} x_{2} z_{2}+y_{2} z_{2},
\end{array}\right) .
$$

Therefore, model (1) at the equilibrium point $K$ has a Hopf bifurcation. By calculations, we have

$$
\begin{gathered}
\alpha_{1}=\left(\begin{array}{l}
1 \\
0 \\
0
\end{array}\right), \quad \alpha_{2}=\left(\begin{array}{c}
\frac{a}{K} \\
\frac{G}{a K} \\
0
\end{array}\right), \quad \alpha_{3}=\left(\begin{array}{l}
0 \\
0 \\
1
\end{array}\right), \\
T=\left(\alpha_{2}, \alpha_{1}, \alpha_{3}\right)=\left(\begin{array}{ccc}
\frac{a}{k} & 1 & 0 \\
\frac{G}{a k} & 0 & 0 \\
0 & 0 & 1
\end{array}\right),
\end{gathered}
$$

where $k=\sqrt{2 G^{2}-a^{4}} / a$.

For model (10), taking the following transformation $\left(\begin{array}{l}x_{1} \\ y_{1} \\ z_{1}\end{array}\right)=T\left(\begin{array}{l}x_{2} \\ y_{2} \\ z_{2}\end{array}\right)$, we have

$$
\dot{\tilde{x_{2}}}=A \widetilde{x_{2}}+B \text {, }
$$

where
From (13),

$$
\begin{aligned}
& \dot{x}_{2}=-k y_{2}+P\left(x_{2}, y_{2}, z_{2}\right), \\
& \dot{y}_{2}=k x_{2}+Q\left(x_{2}, y_{2}, z_{2}\right), \\
& \dot{z}_{2}=a z_{2}+R\left(x_{2}, y_{2}, z_{2}\right),
\end{aligned}
$$

where

$$
\begin{gathered}
P\left(x_{2}, y_{2}, z_{2}\right)=\frac{a}{k} x_{2}^{2}+x_{2} y_{2}+\frac{a k}{G} x_{0} y_{0}-y_{0}+G, \\
Q\left(x_{2}, y_{2}, z_{2}\right) \\
=-\frac{G^{2}+a^{4}}{a^{2} k^{2}} x_{2}^{2}-\frac{a}{k} x_{2} y_{2}-z_{2}^{2} \\
-\left(\frac{a^{2}}{G}+\frac{2 G}{a k}\right) x_{0} y_{0}-a x_{0}+\frac{a^{2}}{G} x_{0}+a F-a^{2}, \\
R\left(x_{2}, y_{2}, z_{2}\right)=\frac{a}{k} x_{2} z_{2}+y_{2} z_{2} .
\end{gathered}
$$




$$
\begin{aligned}
=\frac{1}{2}( & \left.-1-\frac{G^{2}+a^{4}}{a^{2} k^{2}}\right) i \\
G_{21}= & \frac{1}{8}\left(\frac{\partial P^{3}}{\partial x_{2}^{3}}+\frac{\partial P^{3}}{\partial x_{2} \partial y_{2}^{2}}+\frac{\partial Q^{3}}{\partial x_{2}^{2} \partial y_{2}}+\frac{\partial Q^{3}}{\partial y_{2}^{3}}\right. \\
& \left.+i\left(\frac{\partial Q^{3}}{\partial x_{2}^{3}}+\frac{\partial Q^{3}}{\partial x_{2} \partial y_{2}^{2}}-\frac{\partial P^{3}}{\partial x_{2}^{2} \partial y_{2}}-\frac{\partial P^{3}}{\partial y_{2}^{3}}\right)\right)=0 .
\end{aligned}
$$

Since the dimension $n=3>2$, we obtain the following equations:

$$
\begin{aligned}
& h_{11}=\frac{1}{4}\left(\frac{\partial R^{2}}{\partial x_{2}^{2}}+\frac{\partial R^{2}}{\partial y_{2}^{2}}\right)=0, \\
& h_{20}=\frac{1}{4}\left(\frac{\partial R^{2}}{\partial x_{2}^{2}}-\frac{\partial R^{2}}{\partial y_{2}^{2}}-2 \frac{\partial R^{2}}{\partial x_{2} \partial y_{2}}\right)=0 .
\end{aligned}
$$

Next, we solve the following equations:

$$
\begin{gathered}
\lambda_{1} \phi_{11}=-h_{11}, \\
\left(\lambda_{1}-2 k i\right) \phi_{20}=-h_{20} .
\end{gathered}
$$

Then,

$$
\begin{aligned}
& \phi_{11}=0, \\
& \phi_{20}=0 .
\end{aligned}
$$

Let

$$
\begin{aligned}
& G_{110}=\frac{1}{2}\left(\frac{\partial P^{2}}{\partial x_{2} \partial z_{2}}+\frac{\partial Q^{2}}{\partial y_{2} \partial z_{2}}+i\left(\frac{\partial P^{2}}{\partial x_{2} \partial z_{2}}-\frac{\partial Q^{2}}{\partial y_{2} \partial z_{2}}\right)\right)=0 \\
& G_{101}=\frac{1}{2}\left(\frac{\partial P^{2}}{\partial x_{2} \partial z_{2}}-\frac{\partial Q^{2}}{\partial y_{2} \partial z_{2}}+i\left(\frac{\partial P^{2}}{\partial x_{2} \partial z_{2}}+\frac{\partial Q^{2}}{\partial y_{2} \partial z_{2}}\right)\right)=0 .
\end{aligned}
$$

Taking $m=\left(G^{2}+a^{4}\right) / a^{2} k^{2}$, it then follows from (17) and (21) that

$$
g_{21}=G_{21}+\left(2 G_{110} \phi_{11}+G_{101} \phi_{20}\right)=0 .
$$

Also, let

$$
\begin{aligned}
M_{1}(0) & =\frac{i}{2 k}\left(g_{20} g_{11}-2\left|g_{11}\right|^{2}-\frac{1}{3}\left|g_{02}\right|^{2}\right)+\frac{1}{2} g_{21} \\
& =\frac{(m+1) a}{8 k^{2}}-\frac{i}{8 k}\left(\frac{2 a^{2}}{k^{2}}+\frac{10}{3} m^{2}+\frac{1}{3} m+\frac{1}{3}\right) .
\end{aligned}
$$

Then, we have

$$
\begin{gathered}
\mu_{2}(a, b)=-\frac{\operatorname{Re}\left(M_{1}(0)\right)}{\alpha^{\prime}(0)}=-\frac{(m+1) a}{4 k^{2}}>0, \quad(a<0), \\
\beta_{2}(a, b)=2 \operatorname{Re}\left(M_{1}(0)\right)=\frac{(m+1) a}{4 k^{2}}<0, \quad(a<0), \\
\tau_{2}(a, b)=-\frac{\operatorname{Im}\left(M_{1}(0)\right)+\mu_{2}(a, b) \omega^{\prime}(0)}{b} \\
=-\left(\frac{1}{8 k^{2}}\left(\frac{2 a^{2}}{k^{2}}+\frac{10}{3} m^{2}+\frac{1}{3} m+\frac{1}{3}\right)\right. \\
\left.-\frac{(m+1) a}{16 k^{2}} \frac{\sqrt{4 G^{2}-a^{4}}}{4\left(2 G^{2}-a^{4}\right)}\right)<0 .
\end{gathered}
$$

Theorem 3. When $2 G^{2}>a^{4}, a<0$, there is a Hopf bifurcation in model (1) at $x_{0}=a+1$, and

(1) $\mu_{2}(a, b)>0$; that is to say, the direction of bifurcation is supercritical,

(2) $\beta_{2}(a, b)<0$; that is to say, the solutions of bifurcating periodic solutions are orbitally stable,

(3) $\tau_{2}(a, b)<0$; that is to say, the periods of bifurcating periodic solutions increase.

Proof. It is easy to obtain the result from the above derivative process in Section 2.3 and is therefore omitted here.

2.4. The Stability and Bifurcation Analysis with Parameters $G \neq 0$ and $b \neq 0$. In this section, we consider the stability and bifurcation analysis of model (1) when $G \neq 0$ and $b \neq 0$. Without loss of generality, we first discuss the stability and bifurcation analysis of model (1) at two special equilibrium points when $x=0$ and $x=1$. It is easy to verify that when $x=0$ and $x=1$, the equilibrium points of model (1) are $K_{1}(0, G, 0)$ and $K_{2}(1,0, G / b)$, respectively.

In the following, let us consider the stability and bifurcation analysis of model (1) at the equilibrium point $K_{1}$.

Theorem 4. For model (1), if $a>0$, the equilibrium point $K_{1}$ is stable, and

(1) when $a \geq 1+2 \sqrt{2} G>0$ or $0<a \leq 1-2 \sqrt{2} G$, the equilibrium point $K_{1}$ is stable node point,

(2) when $0<1-2 \sqrt{2} G<a<1+2 \sqrt{2} G$, the equilibrium point $K_{1}$ is stable focus point.

Proof. For model (1), the Jacobian matrix at the equilibrium point $K_{1}$ is

$$
J=\left(\begin{array}{ccc}
-a & -2 G & 0 \\
G & -1 & 0 \\
b G & 0 & -1
\end{array}\right),
$$

and its characteristic equation is

$$
f(\lambda)=(\lambda+1)\left(\lambda^{2}+(a+1) \lambda+a+2 G^{2}\right) .
$$


We can get $\lambda_{1,2,3}$

$$
\begin{gathered}
\lambda_{1}=-1, \\
\lambda_{2,3}=\frac{-(a+1) \pm \sqrt{(a+1)^{2}-4\left(a+2 G^{2}\right)}}{2} .
\end{gathered}
$$

Since $a>0$, it then follows from (27) that $\operatorname{Re}\left(\lambda_{1,2,3}\right)<0$. Therefore, the equilibrium point $K_{1}$ is stable. In the same time, note that if $\Delta=(a+1)^{2}-4\left(a+2 G^{2}\right)>0, \lambda_{1,2,3}<0$ are real numbers, and it then follows that the equilibrium point $K_{1}$ is stable node point; if $\Delta=(a+1)^{2}-4\left(a+2 G^{2}\right)<$ $0, \lambda_{2,3}$ has conjugate imaginary roots, it then follows that the equilibrium point $K_{1}$ is stable focus point. The proof is completed.

Theorem 5. For model (1), if $a<0$ and

(1) when $a=-1, F<-1 / 2$ and $G^{2}>1 / 2$, then model (1) at the equilibrium point $K_{1}$ has a Hopf bifurcation,

(2) when $a=-1, F=-2$ and $G^{2}=2$, then model (1) at the equilibrium point $K_{1}$ has a Fold bifurcation,

(3) when $a=-1, F=-1 / 2$, then model (1) at the equilibrium point $K_{1}$ has a flip bifurcation.

Proof. According to (27), we can easily get the conclusion; therefore, we omit it here.

Next, let us consider the equilibrium point $K_{2}(1,0, G / b)$.

Theorem 6. If $a>0$ and $F<3 / 2$, then the equilibrium point $K_{2}$ of model (1) is stable.

Proof. The Jacobian matrix of model (1) at the equilibrium point $K_{2}$ is

$$
J=\left(\begin{array}{ccc}
-a & -2 G & -2 \frac{G}{b} \\
G & 0 & b \\
-\frac{G}{b} & b & 0
\end{array}\right)
$$

and its characteristic equation is

$$
f(\lambda)=\lambda^{3}+a \lambda^{2}+\left(b^{2}+\frac{2 G^{2}}{b^{2}}\right) \lambda+a b^{2}-2 G^{2} .
$$

Let $A=a, B=b^{2}+\left(2 G^{2} / b^{2}\right)$, and $C=a b^{2}-2 G^{2}$. By the Routh-Hurwitz criterion, when $A>0, C>0$ and $A B-C>0$, the eigenvalues of (29) have negative real parts. Therefore, we can get $A B-C=2 G^{2}\left(1+\left(a / b^{2}\right)\right)>0, C>0$, and $F<3 / 2$. Therefore, the equilibrium point $K_{2}$ of model (1) is stable. The proof is completed.

In the above discussions, we consider the stability and bifurcation analysis of model (1) at two special equilibrium points $K_{1}$ and $K_{2}$, respectively. In the following, we will discuss stability and bifurcation analysis of model (1) at the general equilibrium point $K\left(x_{0}, y_{0}, z_{0}\right)$.

Theorem 7. Suppose $K\left(x_{0}, y_{0}, z_{0}\right)$ is an equilibrium point in model (1), if $x_{0}$ is a solution of the equation $a(F-x)(1-2 x+$ $\left.\left(b^{2}+1\right) x^{2}\right)=G^{2}$ and satisfies the inequations

$$
\begin{gathered}
x_{0}<1+\frac{a}{2}, \\
x_{0}^{2}+\frac{4+2 F+2 F b^{2}}{3\left(1+b^{2}\right)} x_{0}+\frac{1+2 F}{3\left(1+b^{2}\right)}>0, \\
x_{0}^{3}-\frac{5 a+2 b^{2}-a b^{2}+6}{2\left(1+b^{2}\right)} x_{0}^{2}+\frac{9 a+6+a F+3 a^{2}-a b^{2} F}{2\left(1+b^{2}\right)} x_{0} \\
-\frac{(1+a)(2+2 a+a F)}{2\left(1+b^{2}\right)}<0,
\end{gathered}
$$

then the equilibrium point $K$ is stable.

Proof. From (2),

$$
\begin{gathered}
y_{0}=g\left(x_{0}, b\right)\left(1-x_{0}\right) G, \\
z_{0}=g\left(x_{0}, b\right) x_{0} b G, \\
a(F-x) f\left(x_{0}, b\right)=G^{2},
\end{gathered}
$$

where $f\left(x_{0}, b\right)=1-2 x_{0}+\left(b^{2}+1\right) x_{0}^{2}$ and $g\left(x_{0}, b\right)=1 / f\left(x_{0}, b\right)$. The Jacobian matrix of model (1) at the point $K\left(x_{0}, y_{0}, z_{0}\right)$ is given as

$$
J(0)=\left(\begin{array}{ccc}
-a & -2 g\left(x_{0}, b\right)\left(1-x_{0}\right) G & -2 g\left(x_{0}, b\right) b x_{0} G \\
g\left(x_{0}, b\right)\left(1-x_{0}-b^{2} x_{0}^{2}\right) G & x_{0}-1 & -b x_{0} \\
b g\left(x_{0}, b\right) G & b x_{0} & x_{0}-1
\end{array}\right)
$$


Taking $G g\left(x_{0}, b\right)=T$, we can get

$$
\begin{aligned}
f(\lambda)= & \lambda^{3}+\left(2-2 x_{0}+a\right) \lambda^{2} \\
& +\left(2 a\left(1-x_{0}\right)+f\left(x_{0}, b\right)\left(1+2 T^{2}\right)\right) \lambda \\
& +f\left(x_{0}, b\right)\left(a+2 T^{2}\left(1-x_{0}-b^{2} x_{0}\right)\right) .
\end{aligned}
$$

Let $A=2-2 x_{0}+a, B=2 a\left(1-x_{0}\right)+f\left(x_{0}, b\right)\left(1+2 T^{2}\right)$, and $C=f\left(x_{0}, b\right)\left(a+2 T^{2}\left(1-x_{0}-b^{2} x_{0}\right)\right)$.

Note that the $f\left(x_{0}, b\right)>0, g\left(x_{0}, b\right)>0, T^{2}>0$, and

$$
\begin{gathered}
x_{0}<1+\frac{a}{2}, \\
x_{0}^{2}+\frac{4+2 F+2 F b^{2}}{3\left(1+b^{2}\right)} x_{0}+\frac{1+2 F}{3\left(1+b^{2}\right)}>0, \\
x_{0}^{3}-\frac{5 a+2 b^{2}-a b^{2}+6}{2\left(1+b^{2}\right)} x_{0}^{2} \\
+\frac{9 a+6+a F+3 a^{2}-a b^{2} F}{2\left(1+b^{2}\right)} x_{0} \\
-\frac{(1+a)(2+2 a+a F)}{2\left(1+b^{2}\right)}<0,
\end{gathered}
$$

and we have $A>0, C>0$, and $A B-C>0$. According to the Routh-Hurwitz criterion, the real parts of the roots of (33) are all negative. Therefore, the equilibrium point $K$ is stable. The proof is completed.

Theorem 8. Suppose that $K\left(x_{0}, y_{0}, z_{0}\right)$ is an equilibrium point in model (1). If $x_{0}$ is a solution of the equation $a(F-x)(1-$ $\left.2 x+\left(b^{2}+1\right) x^{2}\right)=G^{2}$ and satisfies the following inequations and equation:

$$
\begin{gathered}
x_{0}<1+\frac{a}{2}, \\
x_{0}^{2}+\frac{4+2 F+2 F b^{2}}{3\left(1+b^{2}\right)} x_{0}+\frac{1+2 F}{3\left(1+b^{2}\right)}>0, \\
x_{0}^{3}-\frac{5 a+2 b^{2}-a b^{2}+6}{2\left(1+b^{2}\right)} x_{0}^{2} \\
+\frac{9 a+6+a F+3 a^{2}-a b^{2} F}{2\left(1+b^{2}\right)} x_{0} \\
-\frac{(1+a)(2+2 a+a F)}{2\left(1+b^{2}\right)}=0, \\
\beta_{1} x_{0}^{4}-\beta_{2} x_{0}^{3}+\beta_{3} x_{0}^{2}+\beta_{4} x_{0}+\beta_{5} \neq 0,
\end{gathered}
$$

then model (1) has a Hopf bifurcation, where

$$
\begin{aligned}
\beta_{1}= & \frac{4 a^{2}\left(1-b^{4}\right)}{G^{2}}, \\
\beta_{2}= & \frac{4 a^{2}}{G^{2}}\left(2+2 b^{2}+a+a b^{2}+2 F-2 F b^{4}\right), \\
\beta_{3}= & \frac{4 a^{2} F^{2}\left(1-b^{4}\right)}{G^{2}}+\frac{8 F a^{2}\left(2+2 b^{2}+a+a b^{2}\right)}{G^{2}} \\
& +3\left(b^{2}+1\right), \\
\beta_{4}= & 4 a b^{2}-14 a-8-4 b^{2}-\frac{4 a^{2} F\left(2+2 b^{2}+a+a b^{2}\right)}{G^{2}}, \\
\beta_{5}= & 12 a+2 a F+2 a b^{2} F+4 a^{2}+3 .
\end{aligned}
$$

Proof. From (33), we can obtain $A=2-2 x_{0}+a, B=2 a(1-$ $\left.x_{0}\right)+f\left(x_{0}, b\right)\left(1+2 T^{2}\right)$, and $C=f\left(x_{0}, b\right)\left(a+2 T^{2}\left(1-x_{0}-b^{2} x_{0}\right)\right)$. Since

$$
\begin{gathered}
x_{0}<1+\frac{a}{2}, \\
x_{0}^{2}+\frac{4+2 F+2 F b^{2}}{3\left(1+b^{2}\right)} x_{0}+\frac{1+2 F}{3\left(1+b^{2}\right)}>0 \\
x_{0}^{3}-\frac{5 a+2 b^{2}-a b^{2}+6}{2\left(1+b^{2}\right)} x_{0}^{2} \\
+\frac{9 a+6+a F+3 a^{2}-a b^{2} F}{2\left(1+b^{2}\right)} x_{0} \\
-\frac{(1+a)(2+2 a+a F)}{2\left(1+b^{2}\right)}=0,
\end{gathered}
$$

it follows that $A\left(x_{0}\right)>0, B\left(x_{0}\right)=C\left(x_{0}\right) / A\left(x_{0}\right)>0$, and $C\left(x_{0}\right)>0$. Similarly, since $\beta_{1} x_{0}^{4}-\beta_{2} x_{0}^{3}+\beta_{3} x_{0}^{2}+\beta_{4} x_{0}+\beta_{5} \neq 0$, it follows that $C^{\prime}\left(x_{0}\right) \neq A^{\prime}\left(x_{0}\right) B\left(x_{0}\right)-A\left(x_{0}\right) B^{\prime}\left(x_{0}\right)$, where

$$
\begin{aligned}
\beta_{1}= & \frac{4 a^{2}\left(1-b^{4}\right)}{G^{2}}, \\
\beta_{2}= & \frac{4 a^{2}}{G^{2}}\left(2+2 b^{2}+a+a b^{2}+2 F-2 F b^{4}\right), \\
\beta_{3}= & \frac{4 a^{2} F^{2}\left(1-b^{4}\right)}{G^{2}}+\frac{8 F a^{2}\left(2+2 b^{2}+a+a b^{2}\right)}{G^{2}} \\
& +3\left(b^{2}+1\right), \\
\beta_{4}= & 4 a b^{2}-14 a-8-4 b^{2} \\
& -\frac{4 a^{2} F\left(2+2 b^{2}+a+a b^{2}\right)}{G^{2}}, \\
\beta_{5}= & 12 a+2 a F+2 a b^{2} F+4 a^{2}+3 .
\end{aligned}
$$


Hence, we have proven that $A\left(x_{0}\right)>0, B\left(x_{0}\right)=$ $C\left(x_{0}\right) / A\left(x_{0}\right)>0, C\left(x_{0}\right)>0$, and $C^{\prime}\left(x_{0}\right) \neq A^{\prime}\left(x_{0}\right) B\left(x_{0}\right)-$ $A\left(x_{0}\right) B^{\prime}\left(x_{0}\right)$. Therefore, according to the Hopf bifurcation theorem [30], model (1) has a Hopf bifurcation. The proof is completed.

2.5. Simulation. In this section, some numerical examples and simulations are presented to illustrate the effectiveness of our theoretical results. Here, we mainly discuss and verify the conditions of Theorems 1, 2, and 3.

Firstly, we verify the effectiveness of Theorem 1 with $a>$ 0 . Taking $a=0.25, F=8$, then the curves of $a(F-$ $x)(1-x)^{2}=G^{2}$ and straight line of $x=1$ are shown in Figure 1(a), in which $x$-axis represents the parameter $G$ and $y$-axis represents $x$ values of the equilibrium point $K(x, G /(1-x), 0)$. Note that when $a=0.25, F=8$, and $G=3$, there are three equilibrium points $A=(-1,1.5,0)$, $B=(7,-0.5,0)$, and $C=(4,-1,0)$, which are shown in Figure 1(a). From Figure 1(b), the converging trajectories for points $B$ and $C$ are Heteroclinic orbits, and they will converge to the equilibrium point $A$. Hence, the equilibrium posits $B$, $C$ are unstable. Take an arbitrary point $D$ (see Figure 1) under the conditions $a=0.25, F=8$, and $G=3$. It is shown from Figure $1(\mathrm{~b})$ that the trajectory starting from $D$ converges to $A$. Therefore, we have verified that the point $A$ is stable.

In addition, we consider $a<0$ in Theorem 1 . Choose $a=-0.25, F=-6$, and then the curves of $a(F-$ $x)(1-x)^{2}=G^{2}$ and straight line of $x=1$ are shown in Figure 2(a). When $a=-0.25, F=-6$, and $G=3$, we can obtain three equilibrium points $A(-2,1,0), B(3,-1.5,0)$, and $C(-5,0.5,0)$, which are shown in Figure 2(a). From Figure 2(b), the converging trajectories for points $B$ and $C$ are heteroclinic orbits, and they will converge to the equilibrium point $A$. Hence, the equilibrium posits $B, C$ are unstable, and the equilibrium posits $A$ is stable.

Next, we verify the effectiveness of Theorems 2 and 3. Take the parameters $G=2, F=-4$, and $a=-1$ which satisfy the Hopf bifurcation conditions $a^{3}(F-a-1)=G^{2}$ and $2 G^{2}>a^{4}$. Figure 3(a) shows the phase plane of model (1) with $G=1.9$, $F=-4$, and $a=-1$. It can be seen from Figure 3(a) that there is a stable limit cycle. Figure $3(\mathrm{~b})$ shows the phase plane of model (1) with $G=2.1, F=-4$, and $a=-1$. It can be seen from Figure 3(b) that there is a stable equilibrium point. Therefore, we have verified that model (1) exists a Hopf bifurcation. In addition, we can calculate $\mu_{2}(a, b)=1 / 7$, according to Theorem 5 , and Hopf bifurcation is supercritical. Also, we can calculate $\beta_{2}(a, b)=-94 / \sqrt{7}<0$, according to Theorem 5 , and the periodic solution is stable.

\section{Chaotic Behavior of the Lorenz-84 Model}

In this section, the chaotic behavior of model (1) with the parameters $G, b, a$, and $F$ are discussed, and the complex dynamic behaviors are analyzed by Lyapunov exponents spectrum, bifurcation diagram, Poincaré section, and power spectrum. Especially, the topological horseshoe is given to rigorous approaches to study chaos in model (1). For the sake of simplicity, the initial condition in model (1) is chosen as $[1,1,1]$.

3.1. Dynamical Behaviors of Model (1) by Varying Parameters $G$ and $b$. This model has been found to be chaotic over a wide range of parameters and has many interesting complex dynamical behaviors by varying parameters $G$ and $b$, respectively. It is well known that Lyapunov exponents measure the exponential rates of divergence or convergence of nearby trajectories in phase space. The dynamical behaviors of model (1) by varying parameters $G$ and $b$ are listed in Table 1.

Dynamical behaviors of model (1) by varying parameter $G$ are first discussed. Suppose that the parameters $a=0.25$, $F=8$, and $b=4$ are fixed. Let the parameter $G$ vary in interval $[0,1.4]$. The Lyapunov exponents spectrum is shown in Figure 4. It is shown from Figure 4 that when $G \in$ $[0.831,1.188]$ and $G \in[0.831,1.188]$, the max Lyapunov exponents are greater than zero; that is to say, model (1) has chaotic state. Figure 5(a) shows the bifurcation diagram of model (1) about the parameter G. Figure 5(b) shows the part of Figure 5(a) with $G>0$. It is shown from Figure 5 that when $G \in[0,1.4]$, model (1) can evolve into chaotic state. Moreover, there are some periodic windows in the chaotic regions. It is shown from Figure 5(a) that the dynamical behaviors of model (1) have the symmetry for the parameter $G$; that is, to say, when the parameters $G>0$ and $G<0$, model (1) has the same dynamical behaviors.

To illustrate the chaotic behavior, the parameter $G$ is chosen as $G=1$. The corresponding phase portrait of chaotic attractor is shown in Figure 6(a) and its spectrum is continuous as shown in Figure 6(b). From Figure 6, model (1) has chaotic state. Next, the topological horseshoe will be used to study chaos in model (1). The topological horseshoe is well recognized as one of the most rigorous approaches to study chaos with computer. It has been successfully applied in many chaotic systems and hyperchaotic systems [32-40]. As a basic and striking result in chaotic dynamics, topological horseshoe provides a powerful tool in the rigorous study of chaos and dynamical systems obtaining the topological entropy, verifying the existence of chaos, showing the structure of chaotic attractors, revealing the mechanism inside chaotic phenomena, and so on. Some definitions and properties about the topological horseshoe [33-36] are given at first.

Let $D$ be a compact connected region of $R^{n}$, and let $D_{i}$, $i=1,2, \ldots, m$ be disjoint compact connected subsets of $D$ homeomorphic to the unit square. Let $f: D \rightarrow R^{n}$ be a piecewise continuous map which is a diffeomorphism on each compact set $D_{i}$.

Definition 9 (see $[33,36])$. For each $D_{i}, 1 \leq i \leq m$, let $D_{i}^{1}$ and $D_{i}^{2}$ be two fixed disjoint compact subsets of $D_{i}$ contained in the boundary $\partial D_{i}$. A connected subset $l$ of $D_{i}$ is said to connect $D_{i}^{1}$ and $D_{i}^{2}$, if $l \cap D_{i}^{1} \neq \varnothing$ and $l \cap D_{i}^{2} \neq \varnothing$.

Definition 10 (see $[33,36]$ ). Let $l \subset D_{i}$ be a connection of $D_{i}^{1}$ and $D_{i}^{2}$. We say that $f(l)$ is acrossing $D_{j}$, if $l$ contains a connected subset $l^{\prime}$ such that $f\left(l^{\prime}\right) \subset D_{j}$ is a connection of $D_{j}^{1}$ and $D_{j}^{2}$. In this case, we denote it by $f \mapsto D_{j}$. Furthermore, if 
TABLE 1: The dynamic behaviors of model (1) about the parameters $G, b, a$, and $F$.

\begin{tabular}{lccc}
\hline $\begin{array}{l}\text { The parameter } G \\
(a=0.25, b=4 \text { and } F=8)\end{array}$ & Dynamic behaviors & $\begin{array}{c}\text { The parameter } b \\
(a=0.25, F=8 \text { and } G=1)\end{array}$ & Dynamic behaviors \\
\hline $0<G<0.277$ & Limit cycle & $0<b<1.07$ & Limit point \\
$0.277 \leq G<0.797$ & Limit cycle period 2 & $1.07 \leq b<1.73$ & Limit cycle \\
$0.797 \leq G<0.801$ & Limit cycle period 4 & $1.73 \leq b<2.53$ & Chaos \\
$0.801 \leq G<0.819$ & Limit cycle period 2 & $2.53 \leq b<3.93$ & Limit cycle \\
$0.819 \leq G<0.831$ & Limit cycle period 4 & $3.93 \leq b<4.16$ & Chaos \\
$0.831 \leq G<1.188$ & Chaos & $4.16 \leq b<5.53$ & Limit cycle \\
$1.188 \leq G<1.212$ & Limit cycle period 4 & $5.53 \leq b<5.9$ & Chaos \\
$1.212 \leq G<1.352$ & Chaos & $5.9 \leq b<8.53$ & Limit cycle \\
$1.352 \leq G<1.364$ & Limit cycle period 2 & $8.53 \leq b<8.60$ & Chaos \\
$1.364 \leq G<1.85$ & Limit point & $8.60 \leq b<10$ & Limit cycle \\
\hline The parameter $a$ & Dynamic behaviors & The parameter $F$ & Dynamic behaviors \\
$(b=4, F=8$ and $G=1)$ & Limit point & $(a=0.25, b=4$ and $G=1)$ & Limit point \\
$0<a<0.134$ & Chaos & $0<F<4.313$ & Limit cycle \\
$0.134 \leq a<0.152$ & Limit cycle & $4.313 \leq F<4.518$ & Chaos \\
$0.152 \leq a<0.207$ & Chaos & $4.518 \leq F<5.159$ & Limit cycle \\
$0.207 \leq a<0.240$ & Limit cycle & $5.159 \leq F<6.948$ & Chaos \\
$0.240 \leq a<0.243$ & Chaos & $6.948 \leq F<8.763$ & Limit cycle \\
$0.243 \leq a<0.280$ & Limit cycle & $8.763 \leq F<15$ & \\
$0.280 \leq a<0.3$ & & &
\end{tabular}

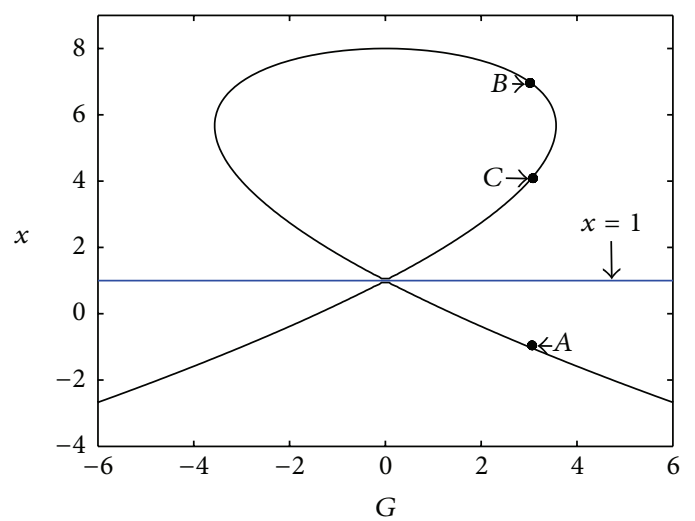

(a)

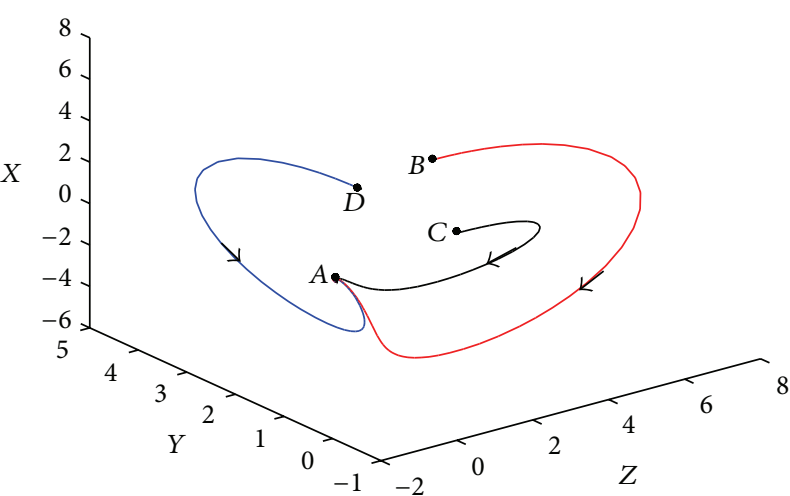

(b)

Figure 1: (a) The curve of $a(F-x)(1-x)^{2}=G^{2}$. (b) Phase plane of system (1) with the parameter $G=3$.

$f \mapsto D_{j}$ holds true for every connection $l$ of $D_{i}^{1}$ and $D_{i}^{2}$, then $f\left(D_{i}\right)$ is said to be acrossing $D_{j}$ and is denoted by $f\left(D_{i}\right) \subset D_{j}$ with respect to two pairs, $\left(D_{i}^{1}, D_{i}^{2}\right)$ and $\left(D_{j}^{1}, D_{j}^{2}\right)$.

Theorem 11 (see [34-36]). If the relation $f\left(D_{i}\right) \subset D_{j}$ holds for every pair with $i, j$, taken from $1 \leq i, j \leq m$, then there exists a compact invariant set $K \subset D$, such that $f \mid K$ is semiconjugate to the full $m$-shift dynamics $\sigma \mid \sum_{m}$ and topological entropy ent $(f) \geq \log m$.

Here, the $m$-shift is also called the Bernoulli $m$-shif. The symbolic series space $\sum_{m}$ is compact, totally disconnected, and perfect. More definitions and properties about the topological horseshoe can be found in [32-40]. To find horseshoes in model (1), we use the method proposed in [34] with an efficient and powerful toolbox in MATLAB called "A toolbox for finding horseshoes in 2D map" (Li Q D. HSTOOL-A MATLAB toolbox for finding horseshoes in 2D maps, 2007-2011. http://www.mathworks.cn/matlabcentral/ fileexchange/14075). When $G=1$, according to the Matlab toolbox, we can get Poincaré map of model (1) at the $z=0$ (Figure $7(\mathrm{a})$ ). We numerically find two quadrilateral $D_{1}$ and $D_{2}$ in the $x-y$ (Poincaré map) plane. The four vertices of $D_{1}$ are

$(0.727230483,1.444887781),(0.666821561,1.360099751)$, $(0.843401487,1.245386534),(0.913104089,1.330174564)$, 


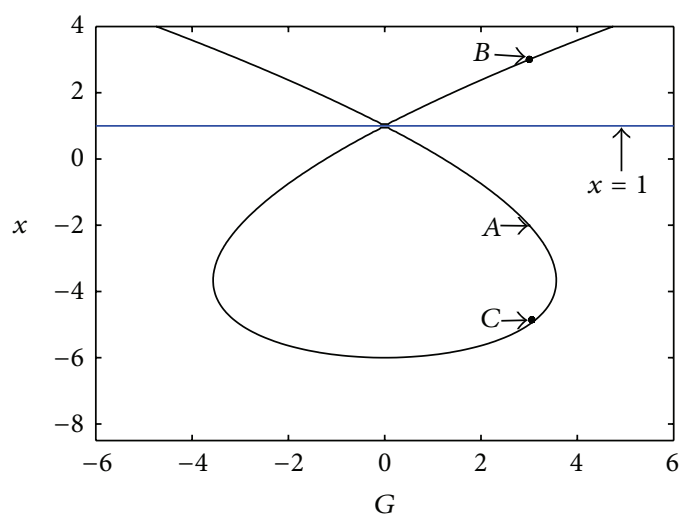

(a)

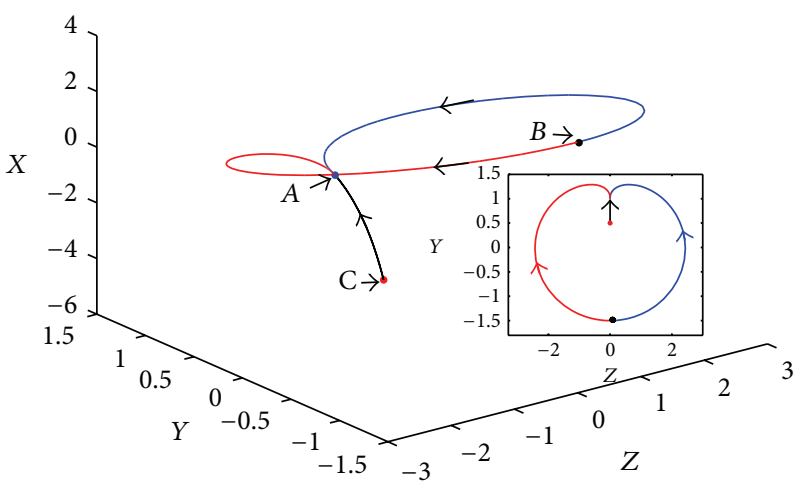

(b)

Figure 2: (a) The curve of $a(F-x)(1-x)^{2}=G^{2}$. (b) Phase plane of of system (1) with the parameter $G=3$.

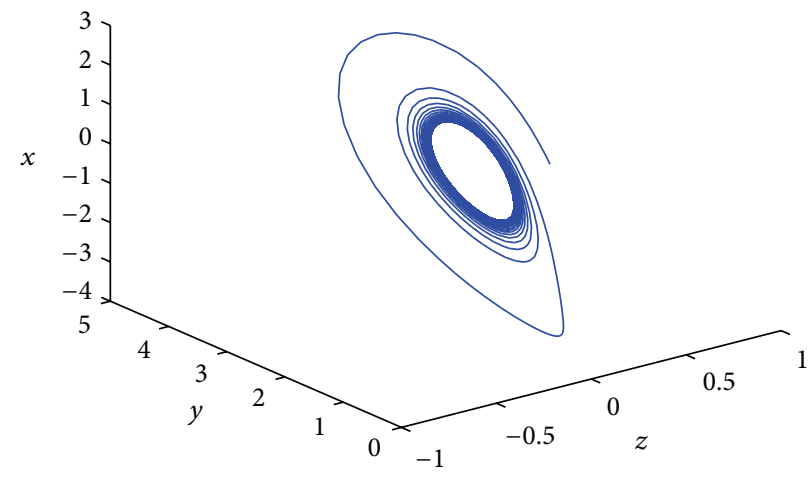

(a)

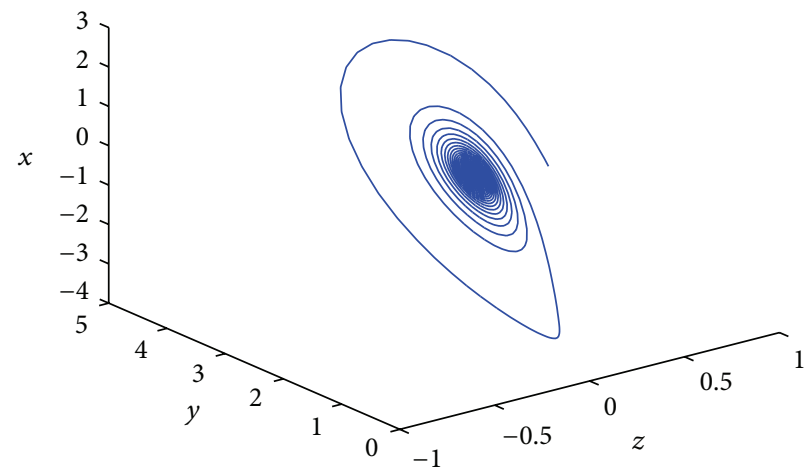

(b)

Figure 3: (a) Phase plane of system (1) with parameter $G=1.9$. (b) Phase plane of system (1) with parameters $G=2.1$.

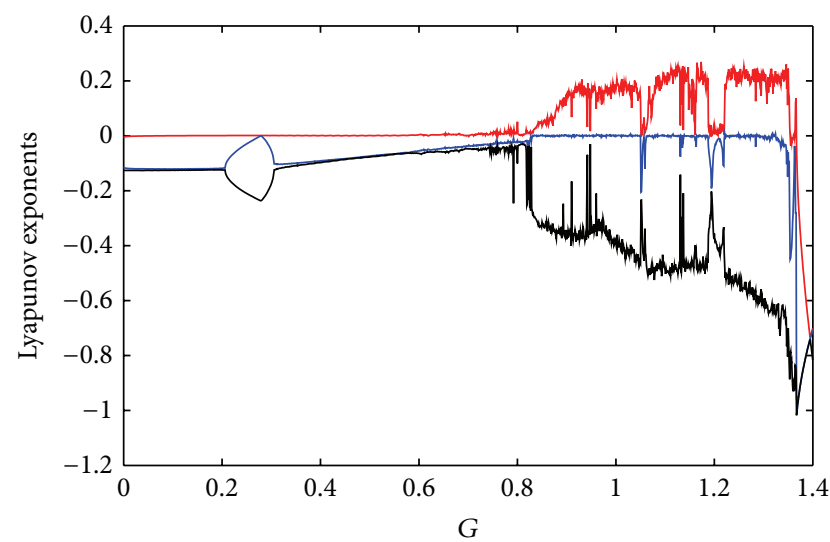

Figure 4: Lyapunov exponents about $G$ for the parameter $F=8$.

and the four vertices of $D_{2}$ are

$(1.029275093,1.245386534),(0.964219331,1.165586035)$,

$(1.085037175,1.040897756),(1.164033457,1.130673317)$.

Based on the theoretical result [32-40] and Theorem 11.
Theorem 12. There exists a compact invariant set $K \subset D_{1} \cup$ $D_{2}$, such that $H^{5} \mid K$ is semiconjugate to 2-shift dynamics and the topological entropy of $H$ is ent $(H) \geq(1 / 5) \log 2$.

Proof. According to Theorem 11, we need to show that the relations

$$
\begin{array}{rlrl}
H^{5}\left(D_{1}\right) & \longmapsto D_{1}, & H^{5}\left(D_{1}\right) \longmapsto D_{2}, \\
H^{5}\left(D_{2}\right) \longmapsto D_{1}, & H^{5}\left(D_{2}\right) \longmapsto D_{2}
\end{array}
$$

hold true. For the above four relations, it is easy to see from Figure 7(b). Furthermore, it also follows from Theorem 11 that the topological entropy of $H$ is $\operatorname{ent}(H) \geq(1 / 5) \log 2$. Clearly, $\operatorname{ent}(H)>0$, which means that model (1) is chaotic indeed when $G=1$. The proof is completed.

In [17], when the parameters $a$ and $b$ are, respectively, chosen as $a=0.25$ and $b=4$, Lorenz pointed out that $F$ and $G$ should be allowed to vary periodically during one year. In particular, $F$ should be larger in winter than in summer. In the numerical study of [17], the author pointed that $(F, G)=(6,1)$ and $(F, G)=(8,1)$ represent the summer conditions and the winter conditions, respectively. With the winter conditions $(F, G)=(8,1)$, model (1) has chaotic 


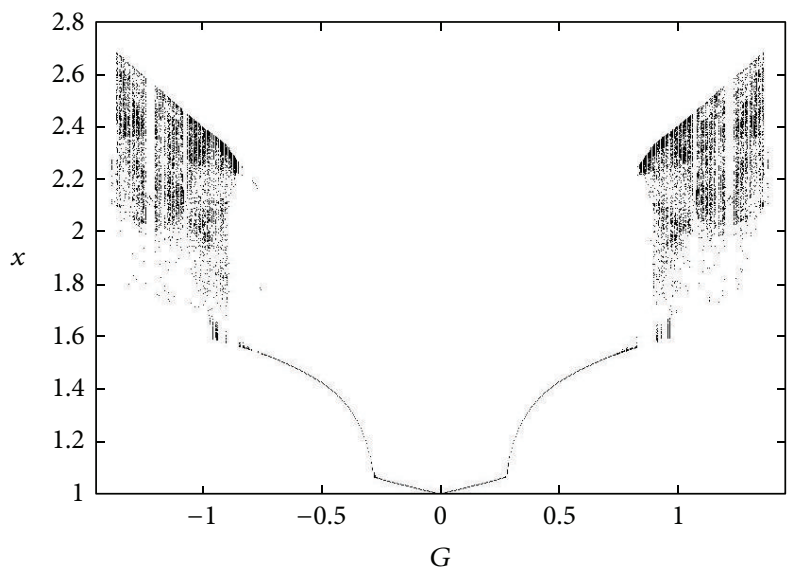

(a)

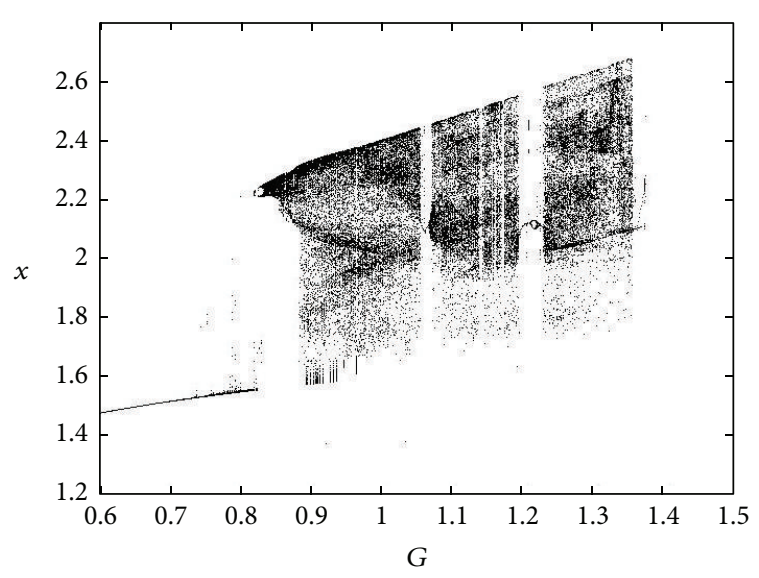

(b)

FIgURE 5: The bifurcation diagram about $G$.

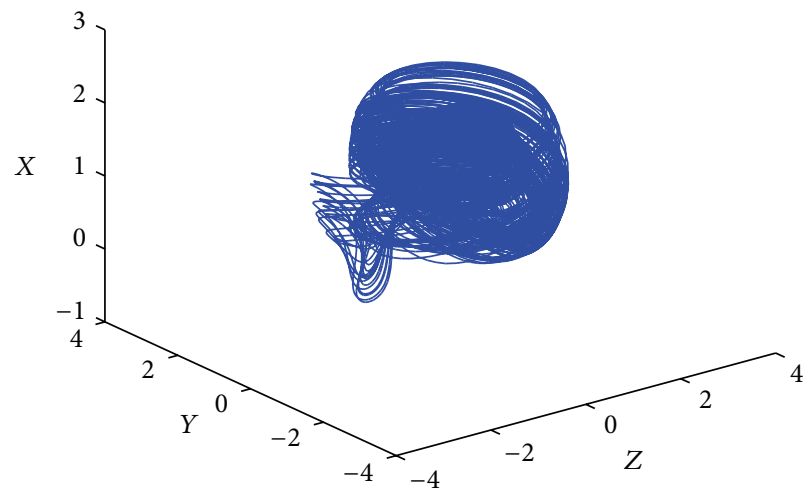

(a)

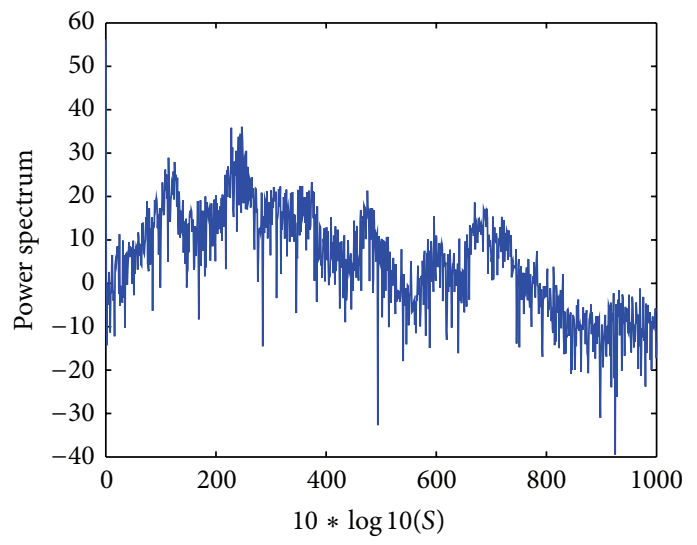

(b)

Figure 6: (a) Phase portraits of the chaos attractor. (b) Spectrum of $x$.

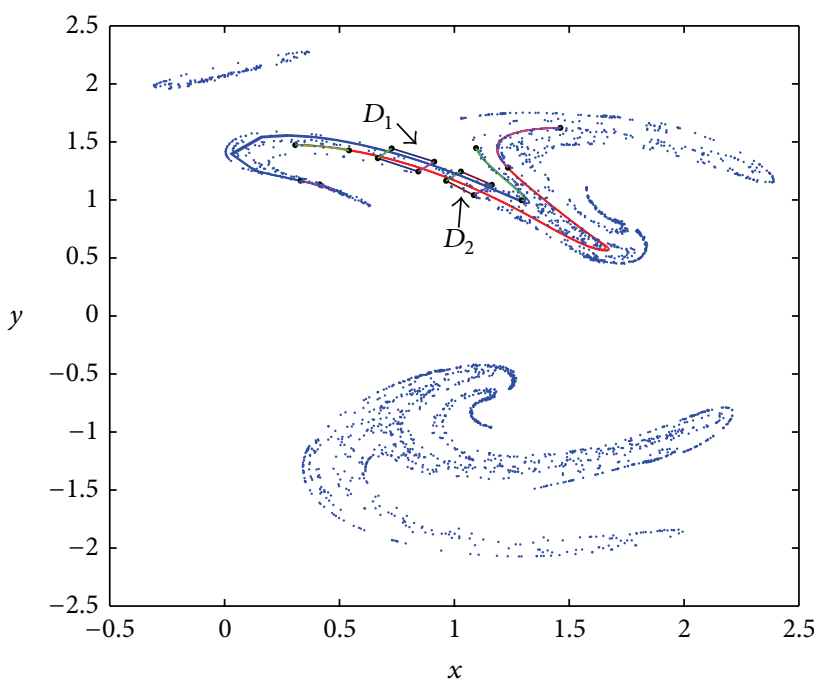

(a)

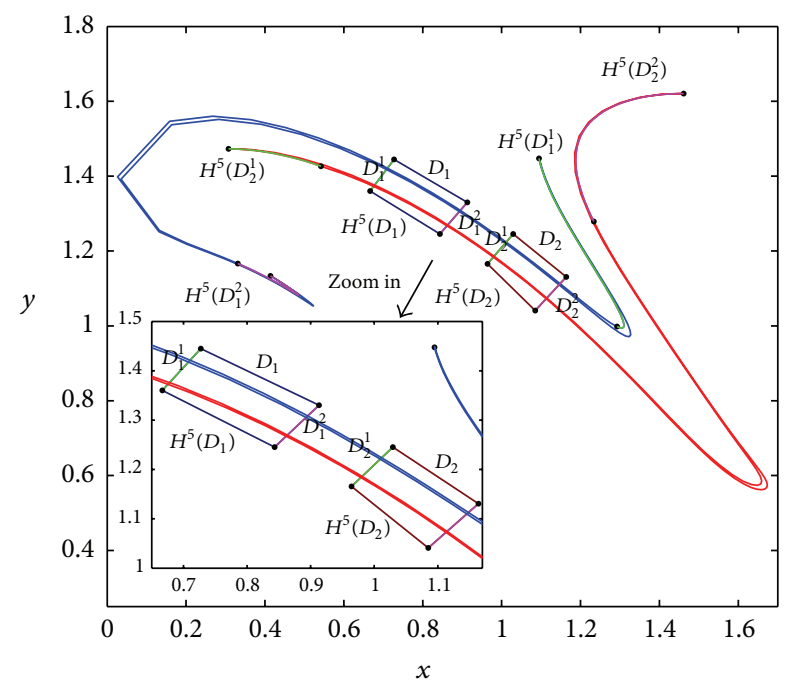

(b)

Figure 7: (a) Poincare mapping on the crossing $z=0$. (b) The topological horseshoe at $G=1$. 


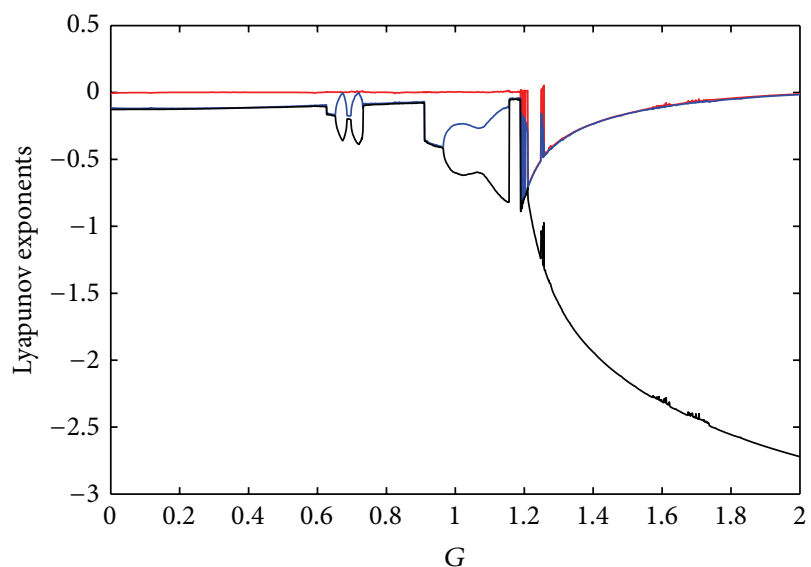

(a) Lyapunov exponents about $G$ for the parameter $F=6$

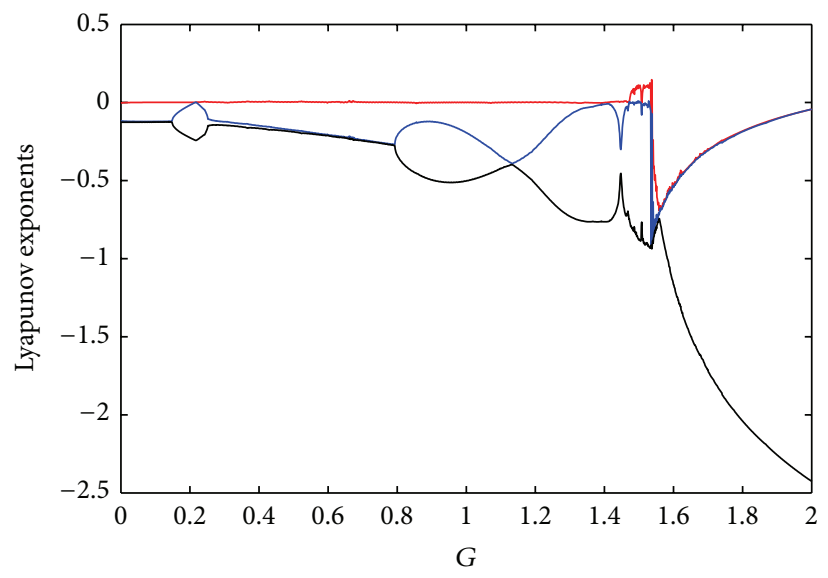

(b) Lyapunov exponents about $G$ for the parameter $F=10$

FIgURE 8: Lyapunov exponents about $G$.

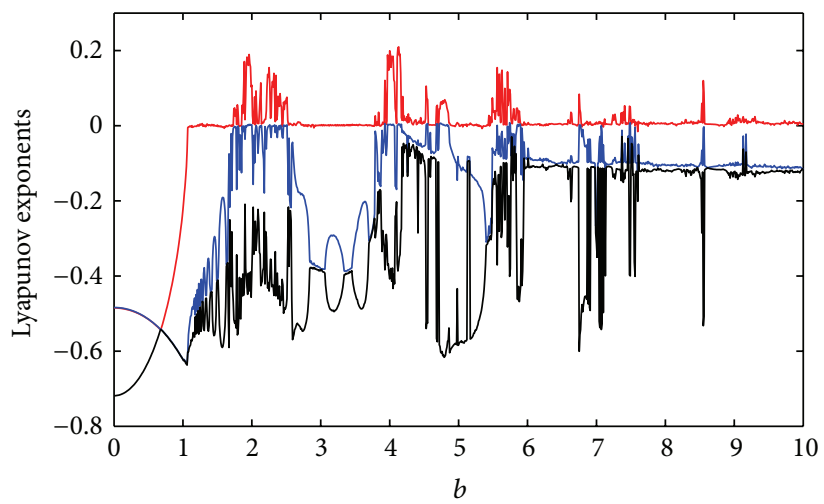

FigURE 9: Lyapunov exponents about $b$.

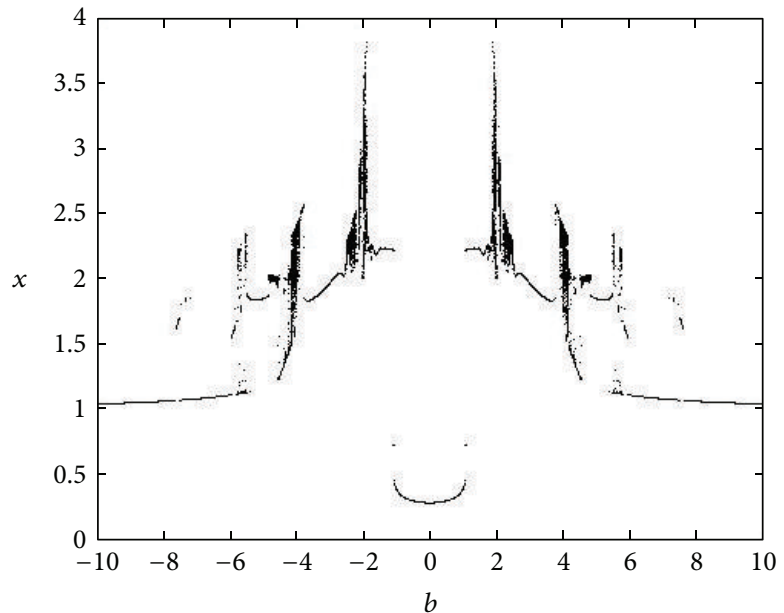

(a)

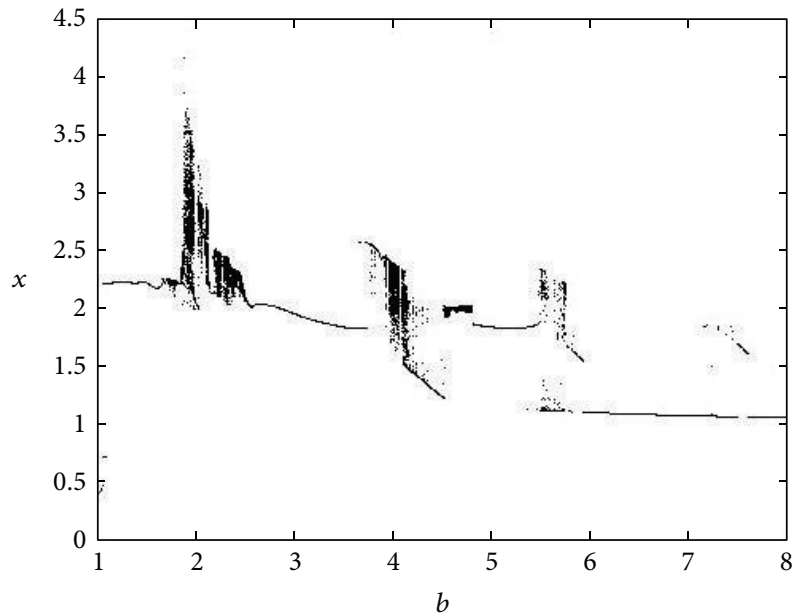

(b)

FIgURE 10: The bifurcation diagram about $b$. 


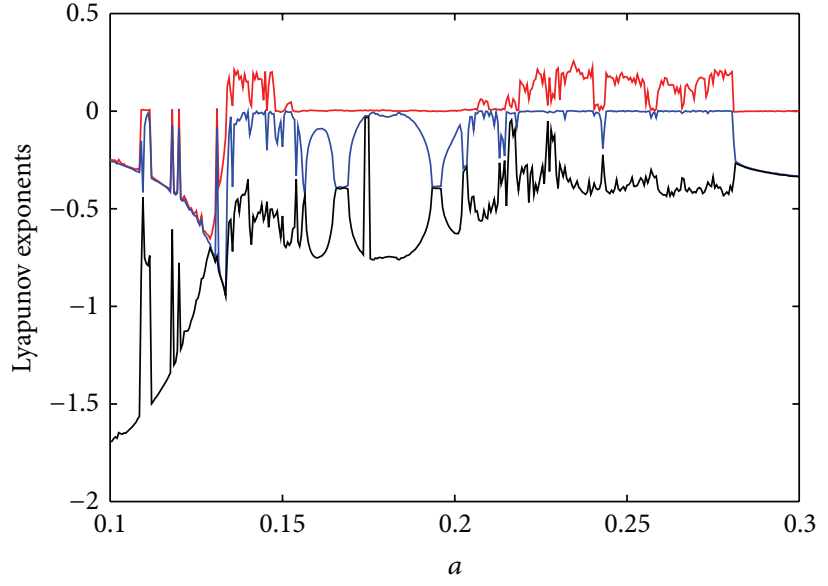

(a)

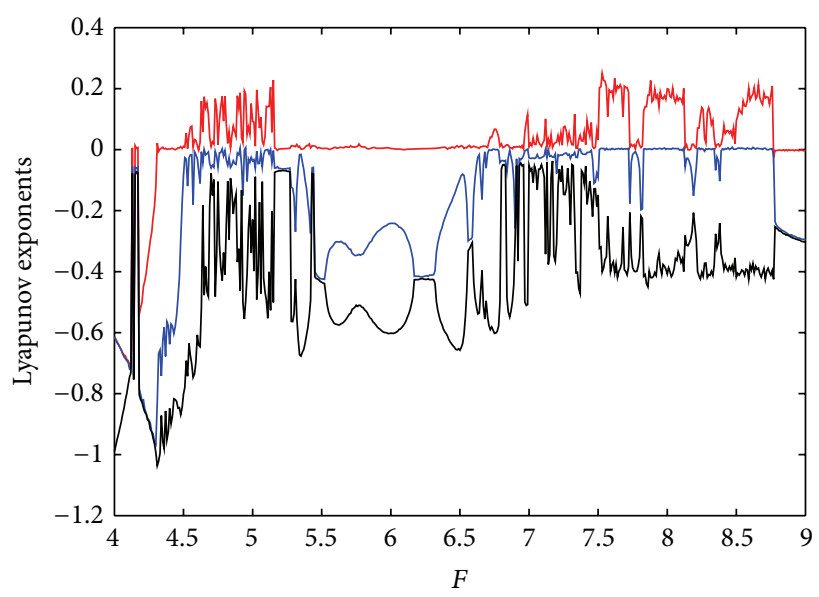

(b)

Figure 11: (a) Lyapunov exponents about $a$. (b) Lyapunov exponents about $F$.

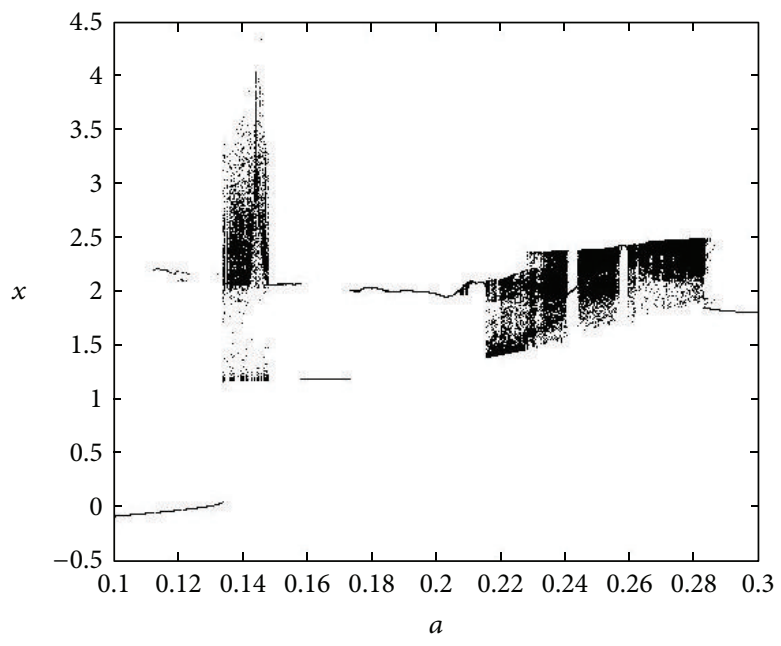

(a)

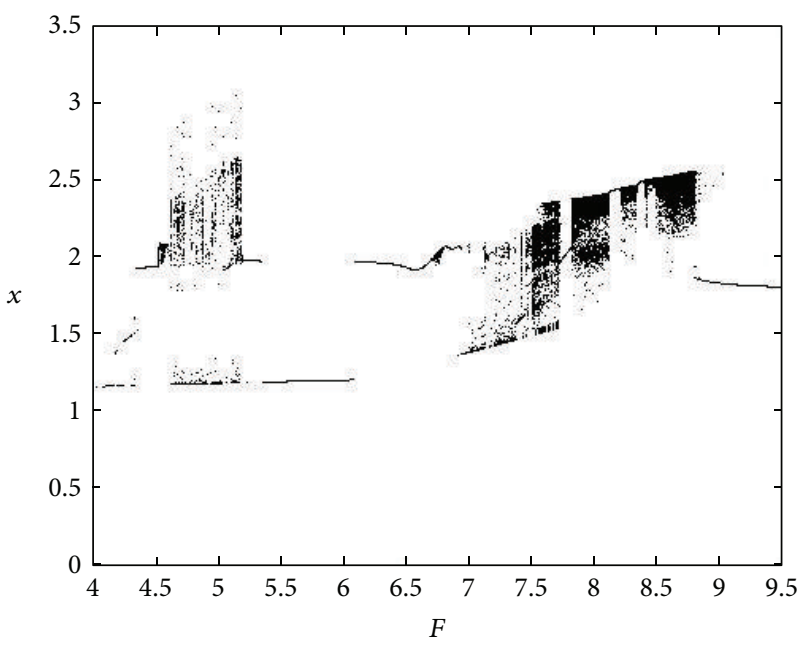

(b)

FIgURE 12: (a) The bifurcation diagram about $a$. (b) The bifurcation diagram about $F$.

behavior. In this paper, $a$ and $b$ are, respectively, chosen as 0.25 and 4 , and $F$ is chosen as $6,7,8,9$, and 10 , respectively. According to the numerical study, model (1) appears to be of more complex dynamics behavior when $F=8$ than when $F=$ $6,7,9,10$ by varying parameter $G$. Moreover, model (1) has been found to be chaotic over more wider range of parameter $G$ when $F=8$ than when $F=6,7,9,10$, respectively. When $F=8$, the Lyapunov exponent spectra have been already shown in Figure 4 . In the following, we consider the cases of $F=6$ and $F=10$, respectively. The cases of $F=7$ and $F=9$ are similar to the cases of $F=6$ and $F=10$, therefore, they are omitted here. When the parameter $F=6$ and $F=10$, the Lyapunov exponents about $G$ are shown in Figures 8(a) and $8(\mathrm{~b})$, respectively. It is shown that from Figure $8(\mathrm{a})$ that model (1) has no chaotic behavior when $F=6$. And from
Figure $8(\mathrm{~b})$, when $F=10$, model (1) has the chaotic behaviors with $1.473 \leq G<1.538$.

Dynamical behaviors of model (1) by varying parameters $b$ are also considered. When parameters $a=0.25, F=8$, and $G=1$ are fixed, let the parameter $b$ vary in interval $[0,10]$. Similarly, the Lyapunov exponents spectrum is shown in Figure 9. It is shown from Figure 9 that when $b \in[1.73,2.53]$, $b \in[3.93,4.16], b \in[5.53,5.90]$, and $b \in[8.53,8.60]$, the max Lyapunov exponents are greater than zero; that is to say, model (1) has chaotic state. The corresponding bifurcation diagram is displayed in Figure 10. It is shown from Figure 10(a) that the dynamical behaviors of model (1) have the symmetry for the parameter $b$; , that is, to say, when the parameter $b>0$ and $b<0$, model (1) has the same dynamical behaviors. Figure 10(b) shows the part of Figure 10(a) with 
$b>0$. This is similar to the parameter $G$, and the dynamical behaviors of model (1) about parameter $b$ are more keenly aware of the change than the parameter $G$.

3.2. Dynamical Behaviors of Model (1) by Varying Parameters $a$ and $F$. This section mainly focuses on the dynamical behaviors of model (1) by varying parameters $a$ and $F$, respectively. The dynamical behaviors of model (1) by varying parameters $a$ and $F$ are also listed in Table 1. Dynamical behaviors of model (1) by varying parameters $a$ are first considered. When parameters $b=4, F=8$, and $G=1$ are fixed, let the parameter $a$ varies in interval $[0.1,0.3]$, the Lyapunov exponents spectrum is shown in Figure 11(a). It is shown from Figure 11(a) that when $a \in[0.1345,0.1526]$, $a \in[0.207,0.2406]$, and $a \in[0.2435,0.2806]$ and the max Lyapunov exponents are greater than zero, model (1) has chaotic state. The corresponding bifurcation diagram is displayed in Figure 12(a).

Similarly, when parameters $a=0.25, b=4$, and $G=1$ are fixed, let the parameter $F$ vary in interval $[4,9]$, the Lyapunov exponents spectrum is shown in Figure 11(b). When $F \in$ $[4.518,5.159]$ and $F \in[6.948,8.763]$, the max Lyapunov exponents are greater than zero, and model (1) has chaotic state. Figure 12(b) shows the bifurcation diagram of model (1) about the parameter $F$. From the Lyapunov exponents spectrum and the bifurcation diagram, shown in Figures 11 and 12, the dynamical behaviors of model (1) about $a$ and $F$ are very similar.

\section{Conclusion}

In this paper, the stability and local bifurcation of the Lorenz84 model have been investigated. The stability and local bifurcation of model (1) with $G \neq 0$ have been discussed. The conditions of the supercritical Hopf bifurcation have also been derived. In addition, the chaotic behaviors of Lorenz84 model have been researched. The bifurcation diagrams and Lyapunov exponents spectrums for every parameter have been discussed and the parameter interval range of limit cycle and chaotic attractor of every parameter have also been calculated. Especially, a computer-assisted proof of the chaoticity of the Lorenz-84 model has been presented by a topological horseshoe theory. Future work will focus on multistability and high codimension bifurcations of the Lorenz-84 model.

\section{Conflict of Interests}

The authors declare that there is no conflict of interests regarding the publication of this paper.

\section{Acknowledgments}

This paper is supported by the National Nature Science Foundation of China (no. 11371049) and Science Foundation of BJTU (2014YJS132).

\section{References}

[1] R. Benzi, A. Sutera, and A. Vulpiani, "The mechanism of stochastic resonance," Journal of Physics A: Mathematical and General, vol. 14, no. 11, pp. L453-L457, 1981.

[2] R. Benzi, G. Parisi, A. Sutera, and A. Vulpiani, "Stochastic resonance in climate change," Tellus, vol. 34, pp. 10-16, 1982.

[3] C. Nicolis, "Solar variability and stochastic effects on climate," Solar Physics, vol. 74, no. 2, pp. 473-478, 1981.

[4] C. Nicolis, "Stochastic aspects of climatic transitions-response to a periodic forcing," Tellus, vol. 34, pp. 1-9, 1982.

[5] C. Nicolis and G. Nicolis, Irreversible Phenomena and Dynamical Systems Analysis in Geosciences, D. Reidel Publishing, 1987.

[6] G. Nicolis and C. Nicolis, Foundations of Complex Systems, World Scientific, Singapore, 2007.

[7] C. Nicolis and G. Nicolis, "Stability, complexity and the maximum dissipation conjecture," Quarterly Journal of the Royal Meteorological Society, vol. 136, no. 650, pp. 1161-1169, 2010.

[8] L. A. Smith, "What might we learn from climate forecasts?" Proceedings of the National Academy of Sciences of the United States of America, vol. 99, no. 1, pp. 2487-2492, 2002.

[9] D. J. Albers and J. C. Sprott, "Structural stability and hyperbolicity violation in high-dimensional dynamical systems," Nonlinearity, vol. 19, no. 8, pp. 1801-1847, 2006.

[10] D. J. Albers, J. C. Sprott, and J. P. Crutchfield, "Persistent chaos in high dimensions," Physical Review E: Statistical, Nonlinear, and Soft Matter Physics, vol. 74, no. 5, Article ID 057201, 2006.

[11] V. Lucarini, A. Speranza, and R. Vitolo, "Parametric smoothness and self-scaling of the statistical properties of a minimal climate model: what beyond the mean field theories?" Physica D: Nonlinear Phenomena, vol. 234, no. 2, pp. 105-123, 2007.

[12] G. Hadley, "On the cause of the general trade winds," Philosophical Transactions of the Royal Society, vol. 34, pp. 58-62, 1735.

[13] A. Persson, "Hadley's principle: understanding and misunderst anding the trade winds," History of Meteorology, vol. 3, pp. 1742, 2006.

[14] F. D. Henry and S. B. Raymond, The Hadley Circulation: Present, Past, and Future, vol. 21 of Advances in Global Change Research, Springer, Amsterdam, The Netherlands, 2004.

[15] D. M. W. Frierson, J. Lu, and G. Chen, "Width of the Hadley cell in simple and comprehensive general circulation models," Geophysical Research Letters, vol. 34, no. 18, Article ID L18804, 2007.

[16] D. J. Seidel, Q. Fu, W. J. Randel, and T. J. Reichler, "Widening of the tropical belt in a changing climate," Nature Geoscience, vol. 1, no. 1, pp. 21-24, 2008.

[17] E. N. Lorenz, "Irregularity: a fundamental property of the atmosphere," Tellus A, vol. 36, no. 2, pp. 98-110, 1984.

[18] E. N. Lorenz, "Can chaos and intransitivity lead to interannual variability?” Tellus, Series A, vol. 42, no. 3, pp. 378-389, 1990.

[19] A. Shil'nikov, G. Nicolis, and C. Nicolis, "Bifurcation and predictability analysis of a low-order atmospheric circulation model," International Journal of Bifurcation and Chaos in Applied Sciences and Engineering, vol. 5, no. 6, pp. 1701-1711, 1995.

[20] H. Broer, C. Simó, and R. Vitolo, "Bifurcations and strange attractors in the Lorenz-84 climate model with seasonal forcing," Nonlinearity, vol. 15, no. 4, pp. 1205-1267, 2002.

[21] V. L. Van, T. Opsteegh, and F. Verhulst, "The dynamics of a low order coupled ocean-atmosphere model," Journal of Nonlinear Science, http://arxiv.org/abs/chao-dyn/9812024. 
[22] P. J. Roebber, "Climate variability in a low-order coupled atmosphere-ocean model," Tellus A, vol. 47, no. 4, pp. 473-494, 1995.

[23] Y. A. Kuznetsov, H. G. E. Meijer, and L. van Veen, “The fold-flip bifurcation," International Journal of Bifurcation and Chaos in Applied Sciences and Engineering, vol. 14, no. 7, pp. 2253-2282, 2004.

[24] Y. G. Yu, "Dynamical analysis of a low-order model representing Hadley circulation," Beijingjiaotong University, vol. 3, pp. 50-54, 2006 (Chinese).

[25] Y. Song, Y. Yu, and H. Wang, "The stability and chaos analysis of the Lorenz-84 atmosphere model with seasonal forcing," in Proceedings of the 4th International Workshop on Chaos-Fractals Theories and Applications (IWCFTA '11), pp. 37-41, October 2011.

[26] L. van Veen, "Baroclinic flow and the Lorenz-84 model," International Journal of Bifurcation and Chaos in Applied Sciences and Engineering, vol. 13, no. 8, pp. 2117-2139, 2003.

[27] L. Niklas, "Bifurcations and strange attractors in a climate related system," Differential Equations and Control Processes, no. 1, pp. 1-53, 2005.

[28] J. G. Freire, C. Bonatto, C. C. DaCamara, and J. A. Gallas, "Multistability, phase diagrams, and intransitivity in the Lorenz-84 low-order atmospheric circulation model," Chaos, vol. 18, no. 3, Article ID 033121, 8 pages, 2008.

[29] V. Pelino and A. Pasini, "Dissipation in Lie-poisson systems and the Lorenz-84 model," Physics Letters A: General, Atomic and Solid State Physics, vol. 291, no. 6, pp. 389-396, 2001.

[30] B. D. Hassard, N. D. Kazarinoff, and Y. Wan, Theory and Applications of Hopf Bifurcation, vol. 41, Cambridge University Press, Cambridge, UK, 1981.

[31] Y. Yu and S. Zhang, "Hopf bifurcation in the Lü system," Chaos, Solitons and Fractals, vol. 17, no. 5, pp. 901-906, 2003.

[32] X.-S. Yang, "Topological horseshoes in continuous maps," Chaos, Solitons \& Fractals, vol. 33, no. 1, pp. 225-233, 2007.

[33] X.-S. Yang, "Topological horseshoes and computer assisted verification of chaotic dynamics," International Journal of Bifurcation and Chaos in Applied Sciences and Engineering, vol. 19, no. 4, pp. 1127-1145, 2009.

[34] Q. Li and X. S. Yang, "A simple method for finding topological horseshoes," International Journal of Bifurcation and Chaos in Applied Sciences and Engineering, vol. 20, no. 2, pp. 467-478, 2010.

[35] Q. Li, X.-S. Yang, and S. Chen, "Hyperchaos in a spacecraft power system," International Journal of Bifurcation and Chaos, vol. 21, no. 6, pp. 1719-1726, 2011.

[36] Q. Li, S. Tang, and X.-S. Yang, "New bifurcations in the simplest passive walking model," Chaos, vol. 23, no. 4, Article ID 043110, 2013.

[37] S. Huan, Q. Li, and X.-S. Yang, "Horseshoes in a chaotic system with only one stable equilibrium," International Journal of Bifurcation and Chaos in Applied Sciences and Engineering, vol. 23, no. 1, Article ID 1350002, 2013.

[38] Q. D. Li and S. Tang, "Algorithm for finding horseshoes in three-dimensional hyperchaotic maps and its application," Acta Physica Sinica, vol. 62, no. 2, Article ID 020510, 2013.

[39] Q. D. Li, S. Tang, and X.-S. Yang, "Hyperchaotic set in continuous chaos-hyperchaos transition," Communications in Nonlinear Science and Numerical Simulation, vol. 19, no. 10, pp. 3718-3734, 2014.
[40] Q. Li and X. S. Yang, "Two kinds of horseshoes in a hyperchaotic neural network," International Journal of Bifurcation and Chaos, vol. 22, no. 8, Article ID 1250200, 2012. 


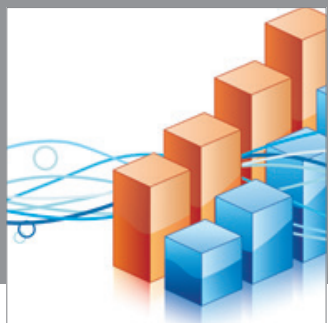

Advances in

Operations Research

mansans

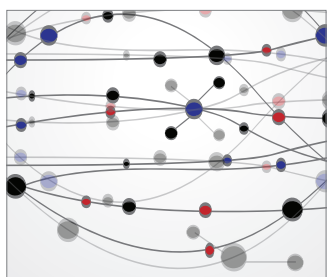

The Scientific World Journal
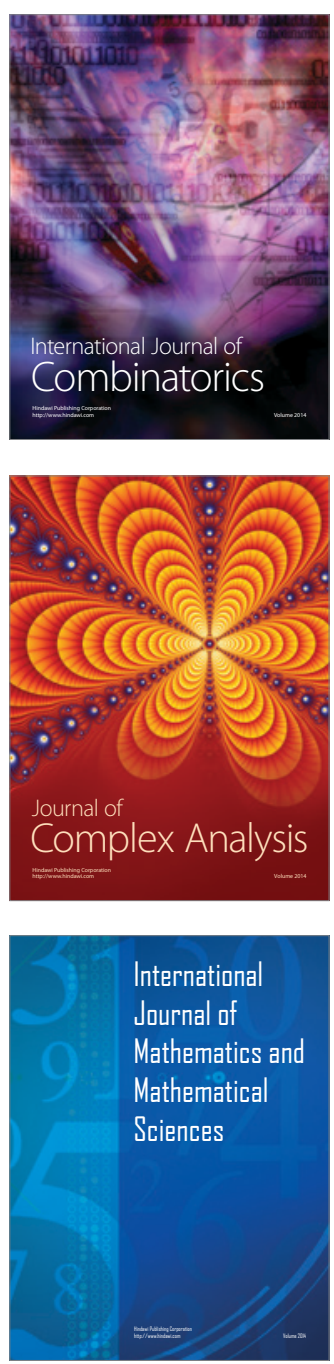
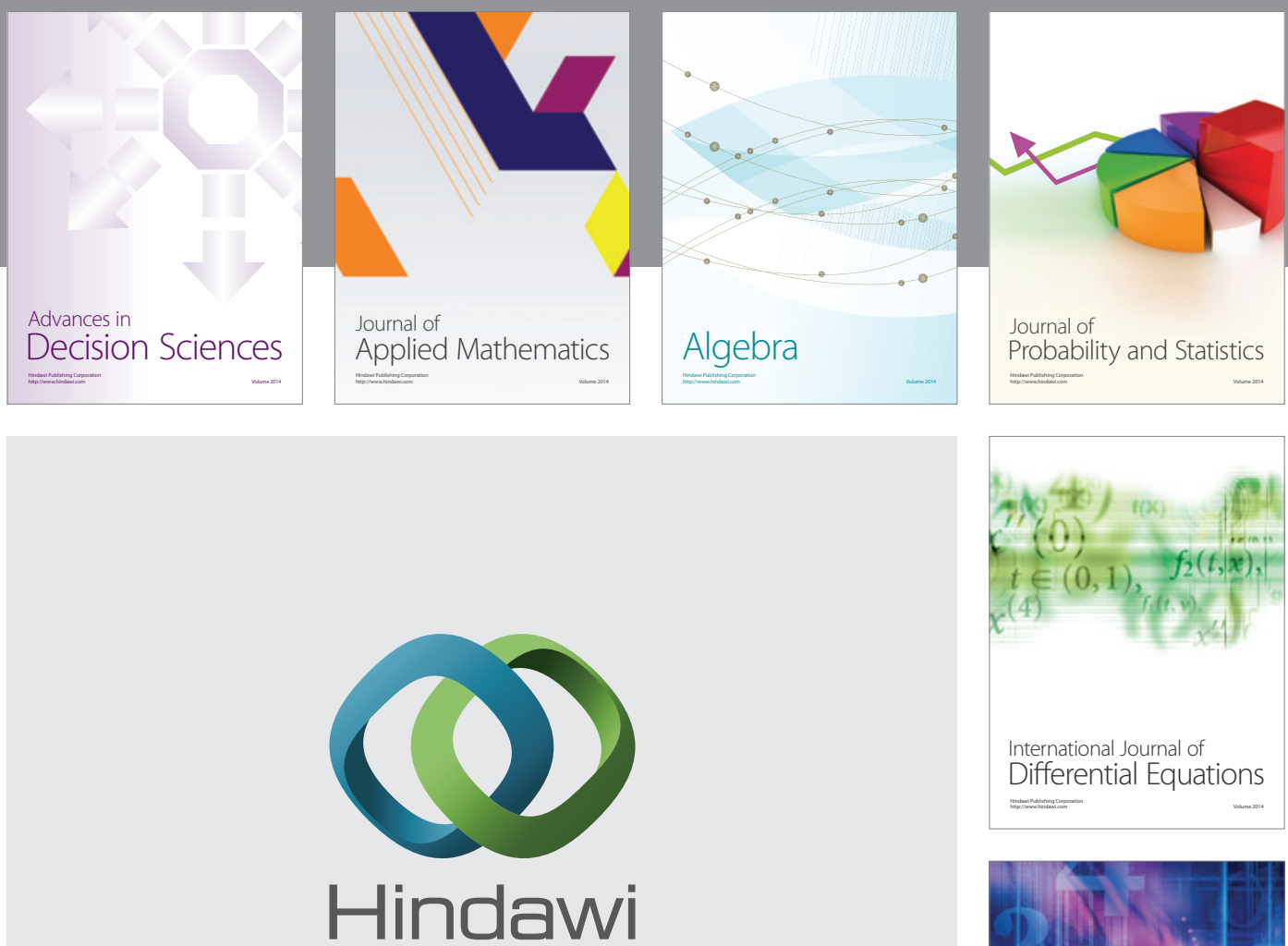

Submit your manuscripts at http://www.hindawi.com
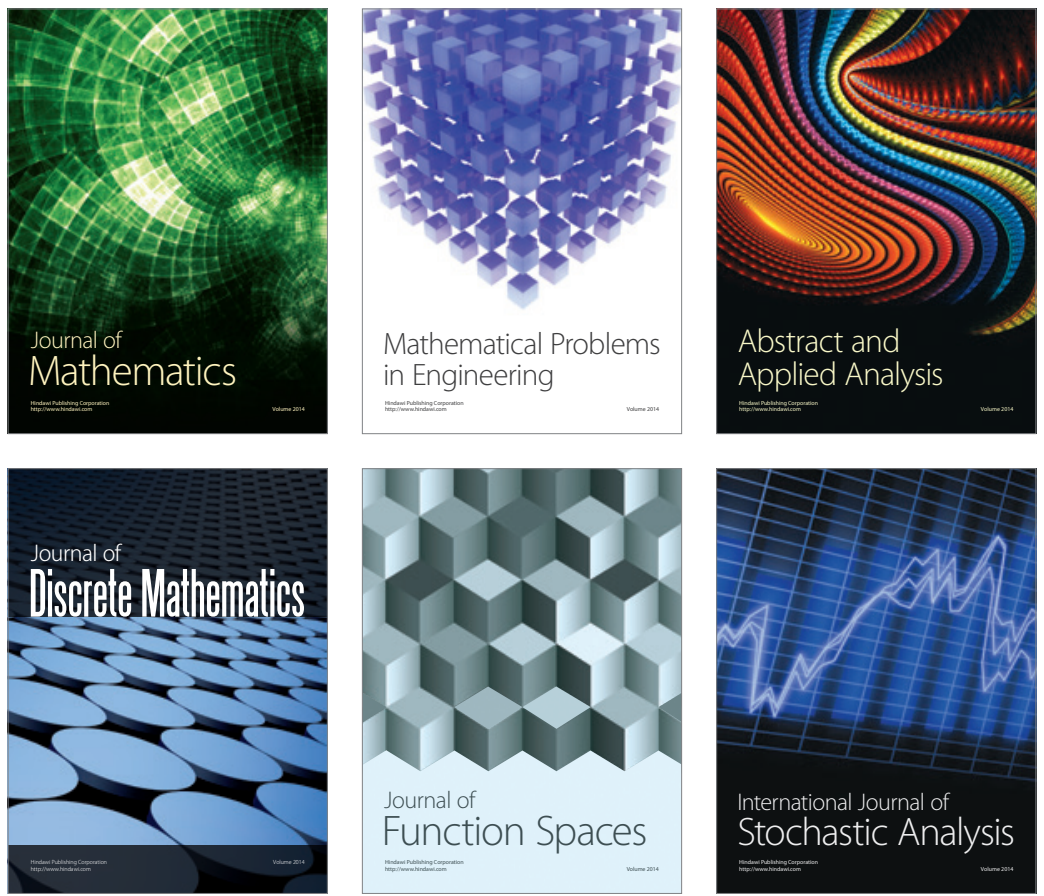

Journal of

Function Spaces

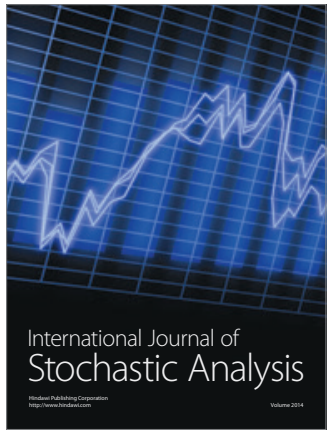

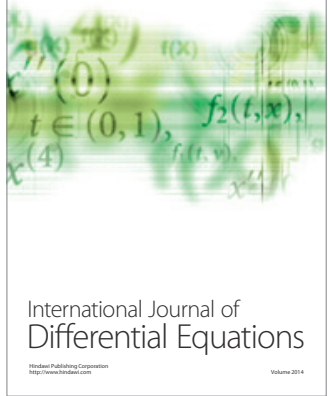
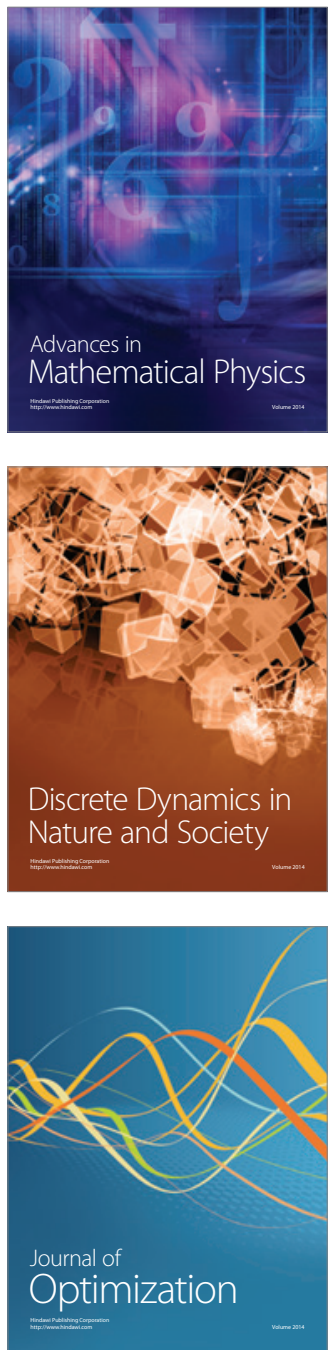